\title{
ADAM17-regulated CX3CL1 expression produced by bone marrow endothelial cells promotes spinal metastasis from hepatocellular carcinoma
}

\author{
CHI SUN ${ }^{*}$, ANNAN HU*, SHENGXING WANG ${ }^{*}$, BO TIAN, LIBO JIANG, \\ YUN LIANG, HOULEI WANG and JIAN DONG
}

Department of Orthopedic Surgery, Zhongshan Hospital, Fudan University, Shanghai 200032, P.R. China

Received September 27, 2019; Accepted March 30, 2020

DOI: 10.3892/ijo.2020.5045

\begin{abstract}
Spinal metastasis occurs in 50-75\% of bone metastases caused by hepatocellular carcinoma (HCC), and HCC-derived spinal metastasis can lead to a less favorable prognosis. Recently, several studies have demonstrated that C-X3-C motif chemokine ligand 1 (CX3CL1) is closely associated with cancer metastasis, and its secretion is modulated by a disintegrin and metalloproteinase 17 (ADAM17). Bone marrow endothelial cells (BMECs) are an essential component of bone marrow. However, little is known about the roles in and effects of BMECs on HCC spinal metastasis. The present study demonstrated that CX3CL1 and C-X-C motif chemokine receptor 3 (CXCR3) expression was upregulated in $\mathrm{HCC}$ spinal metastases, and that CX3CL1 promoted the migration and invasion of HCC cells to the spine. Western blot analysis revealed that the Src/protein tyrosine kinase 2 (PTK2) axis participated in CX3CL1-induced HCC cell invasion and migration. CX3CL1 also increased the expression of M2 macrophage markers in THP-1 monocytes. BMECs promoted the migration and invasion of Hep3B and MHCC $97 \mathrm{H}$ cells by secreting soluble CX3CL1, whereas the neutralization of CX3CL1 inhibited this enhancement. CX3CL1 enhanced the activation of the phosphatidylinositol-4,5-bisphosphate 3-kinase catalytic subunit alpha (PIK3CA)/AKT serine/threonine kinase 1 (AKT1) and Ras homolog family member A (RHOA)/Rho associated coiled-coil containing protein kinase 2 (ROCK2) signaling pathways through the Src/PTK2 signaling pathway. Furthermore, ADAM17 was
\end{abstract}

Correspondence to: Professor Jian Dong, Department of Orthopedic Surgery, Zhongshan Hospital, Fudan University, 180 Fenglin Road, Shanghai 200032, P.R. China

E-mail: dong.jian@zs-hospital.sh.cn

*Contributed equally

Key words: spinal metastasis, hepatocellular carcinoma, bone marrow endothelial cells, C-X3-C motif chemokine ligand 1, a disintegrin and metalloproteinase 17 activated by mitogen-activated protein kinase (MAPK) z14 in BMECs and significantly promoted the secretion of CX3CL1. HCC cells enhanced the recruitment and proliferation of BMECs. The overexpression of CX3CR1 facilitated the spinal metastasis of HCC in a mouse model in vivo. In addition, in vivo experiments revealed that BMECs promoted the growth of HCC in the spine. The present study demonstrated that $\mathrm{CX} 3 \mathrm{CL} 1$ participates in $\mathrm{HCC}$ spinal metastasis, and that BMECs play an important role in the regulation of CX3CL1 in the spinal metastatic environment.

\section{Introduction}

Hepatocellular carcinoma (HCC) accounts for $\sim 90 \%$ of all liver cancer cases, and $\mathrm{HCC}$ has one of the highest mortality rates of all cancer types (1). The prognosis of patients with $\mathrm{HCC}$ has improved in recent years due to therapeutic advancements (2). However, the bone metastases of HCC is becoming a more frequent occurrence (3). The bone is one of the most common sites of HCC metastasis, and the most common site of bone metastases is the spine. Spinal metastases are estimated to account for $50-75 \%$ of HCC bone metastases $(4,5)$. When spinal metastasis occurs, it may result in bone destruction and tumor invasion into the canal space, progressing to axial pain and neurological deficits. Although the prevention and early treatment of HCC with spinal metastasis is an urgent public health concern, there are limited studies available to date on the molecular mechanisms underlying HCC-derived spinal metastasis (6).

Several types of cancer metastasize in an organ-specific manner and organ-specific metastasis is dependent on the interaction of cancer cells with the host microenvironment (7). Tumor cells do not act independently during the establishment of a metastatic microenvironment. The metastatic microenvironment itself provides a suitable environment for the colonization and growth of metastatic cells by expressing several pro-tumorigenic factors. The metastatic cells then regulate the microenvironment by releasing cytokines and interacting with other cells (8). Recently, it was reported that organ-specific endothelial cells regulate organ-specific metastasis (9). Dysregulated endothelial cell behavior is vital in establishing the tumor microenvironment by producing 
several tumor-regulated factors, such as chemokines, that promote angiogenesis, tumor cell survival and metastasis (10). In bone metastasis, communication between endothelial cells and metastatic cells constitutes a vicious cycle, leading to the substantial disruption in the physiological homeostasis of the bone microenvironment. Endothelial cells have been suggested to participate in the tumor metastatic microenvironment, as they constitute a significant part of the spinal vascular system $(11,12)$. However, the focus of studies on bone metastasis has almost always been on osteoblasts and osteoclasts, not endothelial cells. Bone marrow endothelial cells (BMECs) are an important component of the hematopoietic microenvironment that regulate the homing, differentiation, self-renewal and migration of hematopoietic stem cells by releasing several cytokines, including chemokines $(13,14)$. BMECs also regulate immune cells in the bone marrow (15). BMECs, which exert their specific characteristics in the bone marrow microenvironment, have been widely studied in multiple myeloma $(16,17)$. However, the role of BMECs in spinal metastasis has not been extensively studied to date.

Chemokines are a family of small structurally related secreted cytokines, which play essential roles in inflammation and immunity (18). In addition to the involvement of chemokines in immunology and pathogenesis, recent studies have focused on the role of chemokines in modulating several aspects of cancer metastasis. Among the studied chemokines, C-X3-C motif chemokine ligand 1 (CX3CL1) acts as key regulator in spinal metastasis. CX3CL1 is the only member of the CX3C chemokine subfamily, and is involved in regulating the migration, invasion and survival of cancer cells (19). More specifically, the mucin-like domain of CX3CL1 contains a cleavage site that allows metalloproteases to cleave and release the protein in a soluble form (20). It has been reported that CX3CL1 is closely associated with cancer cell metastasis in prostate (21), gastric (22) and breast (23) cancer, as well as in renal (24) and colon carcinoma (25). According to previous studies by the authors, the quantity of CX3CL1 in the cancellous bone of the spine is higher compared with the limbs, and the inhibition of CX3CL1 has been confirmed as an effective strategy to prevent spinal metastasis from breast and prostate cancer in an in vivo model $(26,27)$. However, the role of CX3CL1 in spinal metastasis from HCC has not yet been investigated, at least to the best of our knowledge.

Considering that BMECs are specialized cells with the capacity to release large quantities of cytokines in the spine, and CX3CL1 found in the spine is released from BMECs and leads to an increase in their associated functions, CX3CL1 may promote the invasion and migration of HCC cells and activate the Src/PTK2 signaling pathway in BMECs. Protein tyrosine kinase 2 (PTK2) has been widely studied and enhances in vivo tumorigenesis and metastasis in $\mathrm{HCC}$, as well as cell invasion and migration in vitro $(28,29)$. The occurrence of these phenotypic changes has been determined to be driven by the activation of downstream pathways, such as the RHOA/ROCK2 and PIK3CA/AKT1 signaling pathways $(30,31)$ In the present study, it was demonstrated that CX3CL1 may promote the activation of the phosphatidylinositol-4,5-bisphosphate 3-kinase catalytic subunit alpha (PIK3CA)/AKT serine/threonine kinase 1 (AKT1) and Ras homolog family member A (RHOA)/Rho associated coiled-coil containing protein kinase 2 (ROCK2) signaling pathways via the Src/PTK2 signaling pathway. The specific mechanism used by BMECs to secrete CX3CL1 was determined. A disintegrin and metalloproteinase 17 (ADAM17), which is expressed by BMECs, was activated by mitogen-activated protein kinase (MAPK) and was essential for CX3CL1 secretion. The results of an in vivo experiment revealed that CX3CR1-expressing HCC cells were attracted to the spine by CX3CL1, which was expressed in spinal cancellous bone. To determine the significance of this observation, the malignant capacities of HCC cells mixed with BMECs were determined. Taken together, the results of the present study demonstrate that CX3CL1 is expressed in BMECs and acts as a driving force of $\mathrm{HCC}$ in the spinal metastatic microenvironment.

\section{Materials and methods}

Patients and cell isolation. There were 25 clinical specimens (healthy vertebral bone from 5 patients with fracture surgery, tumor bones and spinal metastases from $15 \mathrm{HCC}$ patients with spinal metastasis, and primary tumors from $5 \mathrm{HCC}$ patients) used in the present study which were obtained from the Department of Orthopedic Surgery, Zhongshan Hospital, Fudan University (Shanghai, China) between July, 2015 and July, 2019. There were 5 cases of spinal fracture $(51.21 \pm 18.57), 5$ cases of HCC (55.29 \pm 13.44 years) and 15 cases of HCC with spinal metastasis $(62.12 \pm 9.69$ years), and all participants were male. All patients provided informed consent and agreed to participate in the study. The present study was approved by the Ethics Committee of Zhongshan Hospital, Fudan University (approval nos. Y2014-185 and Y2019-085). BMECs were isolated from fresh, healthy human bone marrow collected during surgery from 2 patients, a 57-year-old male patient and a 64-year-old male patient. As BMECs exhibit a different sensitivity to trypsin digestion and adaptability to extracellular matrix (ECM), BMECs were purified from other cells after 3 to 4 passages using trypsin digestion. Morphological observation and immunofluorescence staining were performed using p-selectin (cat no. ab6632; Abcam; 1:400) and CD106 (cat. no. ab215380; Abcam; 1:400) to identify BMECs. These cells also tested negative for the mesenchymal stromal cell markers CD117 (cat. no. ab25022; Abcam; 1:400) and STRO-1 (cat. no. ab214086; Abcam; 1:400). The BMECs were maintained in endothelial cell medium containing 10\% fetal bovine serum (FBS) (cat. no. 10099; Thermo Fisher Scientific, Inc.) at $37^{\circ} \mathrm{C}$ with $5 \% \mathrm{CO}_{2}$.

Reagents. Matrigel was obtained from BD Biosciences (cat. no. 3433-005-01). The MAPK14 inhibitor, SB203580, was purchased from Selleck Chemicals. Lipofectamine ${ }^{\circledR} 2000$ was purchased from Invitrogen (cat. no. 11668019; Thermo Fisher Scientific, Inc.). The Src protein inhibitor, bosutinib $(0.5 \mathrm{nmol} / 1$ for $3 \mathrm{~h})$, PTK2 inhibitor, PF562271 $(3.3 \mu \mathrm{mol} / 1$ for $24 \mathrm{~h})$, and PIK3CA inhibitor, LY294002 (10 $\mu \mathrm{mol} / 1$ for $24 \mathrm{~h}$ ), were obtained from Selleck Chemicals. Phorbol 12-myristate 13 -acetate (PMA) $(10 \mathrm{ng} / \mathrm{ml}$ for $24 \mathrm{~h})$ was purchased from Abcam. Other reagents were purchased from EMD Millipore or Sigma-Aldrich (Merck KGaA).

Cell lines and cell culture. THP-1 (SCSP-567), MHCC97H (SCSP-528) and Hep3B (SCSP-5045) cells were purchased 
from The Cell Bank of Type Culture Collection of the Chinese Academy of Sciences. Human umbilical vein endothelial cells (HUVECs) (ATCC ${ }^{\circledR}$ CRL-1730 ${ }^{\mathrm{TM}}$ ) were purchased from the American Type Culture Collection. All cells were maintained in a humidified atmosphere with $5 \% \mathrm{CO}_{2}$ at $37^{\circ} \mathrm{C}$. MHCC97H and Hep3B cells were maintained in high glucose DMEM containing 10\% FBS. Cell culture medium supplements were obtained from Sigma-Aldrich (Merck KGaA). HUVECs were cultured in endothelial cell medium (cat. no. 1001; ScienCell Research Laboratories, Inc.).

Western blot analysis. Total protein was extracted from cells using a lysis buffer with phenylmethanesulfonylfluoride (cat. no. ST505; Beyotime Institute of Biotechnology) and a phosphorylase inhibitor (cat. no. 78445, Thermo Fisher Scientific, Inc.). The protein concentration was determined by the Coomassie Brilliant Blue method. A total of $40 \mu \mathrm{g}$ proteins were loaded on an SDS gel (12\% lower gel and 5\% upper gel), resolved using SDS-PAGE and transferred to nitrocellulose membranes (EMD Millipore). The membranes were blocked with $5 \%$ non-fat milk in TBS containing $0.05 \%$ Tween-20. The membranes were incubated with the primary antibodies overnight at $4^{\circ} \mathrm{C}$ and then incubated with the secondary antibodies (cat.nos. ab97040, ab6940 and ab6566; 1:2,000; Abcam) for $2 \mathrm{~h}$ at room temperature. The anti-matrix metalloproteinase (MMP)13 antibody (cat. no. sc-101564; 1:1,000) was obtained from Santa Cruz Biotechnology, Inc. The anti-p-PTK2 (Tyr397) (cat. no. 8556; 1:1,000), anti-p-PTK2 (Tyr576) (cat. no. 3281; 1:1,000), anti-p-PTK2 (Tyr925) (cat. no. 3284; 1:1,000), anti-total PTK2 (cat. no. 3285; 1:1,000), anti-p-Src (Tyr416) (cat. no. 6943; 1:1,000), anti-Src antibody (cat. no. 2108; 1:1,000) was purchased from Cell Signaling Technology, Inc. The anti-CX3CL1 (cat. no. ab89229; 1:1,000), anti-CX3CR1 (cat. no. ab95620; 1:1,000), anti-ADAM17 (cat. no. ab2051; 1:1,000), anti-p-ADAM17 (Tyr735) (cat. no. ab182630; 1:1,000), anti-p-MAPK14 (Tyr180) (cat. no. ab4822; 1:1,000), anti-MAPK14 (cat. no. ab31828; 1:1,000), anti-RHOA (cat. no. ab187027; 1:1,000), anti-ROCK2 (cat. no. ab125025), anti-NIMA related kinase 2 (NEK2; cat. no. ab227958; 1:1,000), anti-AKT1 (cat. no. ab81283; 1:1,000), anti-p65-nuclear factor (NF)-кB (cat. no. ab16502; 1:1,000), anti-CD163 (cat. no. ab87099; 1:1,000), anti-MRC1 (cat. no. ab188269; 1:1,000), anti-GAPDH (cat. no. ab8245; 1:1,000) and anti- $\beta$-actin antibodies (cat. no. ab8226; $1: 1,000)$ were obtained from Abcam. The enhanced chemiluminescent (ECL) kit was purchased from Abcam (cat. no. ab133406). The experiments were performed 3 times independently. To statistically compare the differences between groups, $\log _{2}$ transformation was applied to normalize the results of densitometry using ImageJ 1.8.0 software (National Institutes of Health).

Immunohistochemistry. Tissues were collected, sectioned into a thickness of $8 \mu \mathrm{m}$, dewaxed in xylene and rehydrated in graded ethanol. Subsequently, tissues were soaked in $3 \% \mathrm{H}_{2} \mathrm{O}_{2}$ for $20 \mathrm{~min}$ to block endogenous peroxidase. Tissues were incubated with anti-CX3CR1 (cat. no. ab95620; 1:2,000) or anti-CX3CL1 antibody (cat. no. ab89229; 1:2,000) (from Abcam) at $4^{\circ} \mathrm{C}$ overnight. Following secondary antibodies (cat. nos. ab150077 and ab150115; 1:2,000; Abcam) incubation, immunoperoxidase staining was performed using the VECTASTAIN Elite ABC kit (cat. no. AK-5001; Vector
Table I. Primers used for RT-qPCR.

Gene $\quad$ Primer sequences

CX3CR1 F: 5'-AGTGTCACCGACATTTACCTCC-3'

R:5'-AAGGCGGTAGTGAATTTGCAC-3'

CX3CL1 F: 5'-GAATTCCTGGCGGGTCAGCACCTCGGCATA-3' R: 5'-AAGCTTTTACAGGGCAGCGGTCTGGTGGT-3'

ADAM17 F: 5'-GTGAGCAGTTTCT CGAACGC-3' R:5'-AGCTTCTCAAGTCGCAGGTG-3'

GAPDH F: 5'-GTCGGTGTGAACGGATTTG-3' R: 5'-TCCCATTCTCAGCCTTGAC-3'

IL6 F: 5'-ACATCGTCGACAAAATCTCTGCA -3' R:5'-AGCCAGTGTCTCCTTGCTGTTT-3'

CXCL8 F: 5'-CAGTGCATAAAGACATACTCC-3' R: 5'-TTTATGAATTCTCAGCCCTC-3'

CCL3 F: 5'-GCTGTGATCTTCAAGACC-3' R:5'-AAGTCTTCGGAGTTTGGG-3'

MMP2 F: 5'-GTTGGCAGTGCAATACCTGA-3' R:5'-GAGCAAAGGCATCATCCACT-3'

MMP8 F: 5'-CCAAAGAGATCACGGTGACA-3' R:5'-GTTGCTGGTTTCCCTGAAAG-3'

PTHLH F: 5'-CAGTGGAGTGTCCTGGTATT-3' R:5'-GATCTCCGCGATCAGATGGT-3'

F, forward; R, reverse; CX3CR1, C-X3-C motif chemokine receptor 1; CX3CR1, C-X3-C motif chemokine ligand 1; ADAM17, a disintegrin and metalloproteinase 17. IL-6, interleukin-6; CXCL8, chemokine C-X-C motif ligand 8; CCL3, C-C motif chemokine ligand 3; MMP2, matrix metallopeptidase 2; MMP8, matrix metallopeptidase 8; PTHLH, parathyroid hormone like hormone.

Laboratories, Inc.) and 3,3'-diaminobidine-tetrachloric acid as a chromogenic agent. The sections were observed using an optical microscope (Olympus-IX51; Olympus Corporation).

Reverse transcription-quantitative PCR (RT-qPCR). TRIzol ${ }^{\circledR}$ reagent was used to extract total RNA and a Thermo Script RT-PCR system was used to reverse transcribe the RNA into cDNA (cat. no. 12594100; Invitrogen; Thermo Fisher Scientific, Inc.). SYBR Premix Ex Taq ${ }^{\mathrm{TM}}$ II (Takara Bio, Inc.) was used to measure mRNA expression, and $G A P D H$ was used as the endogenous reference gene. Primers were purchased from Sangon Biotech, Co., Ltd. and the sequences of the primers are presented in Table I. The thermocycling conditions for PCR amplification were as follows: Denaturation for $2 \mathrm{~min}$ at $95^{\circ} \mathrm{C}$; followed 30 cycles of $94^{\circ} \mathrm{C}$ for $20 \mathrm{sec}, 58^{\circ} \mathrm{C}$ for $20 \mathrm{sec}$, and $72^{\circ} \mathrm{C}$ for $30 \mathrm{sec}$. The expression ratio was calculated using the $2^{-\Delta \Delta \mathrm{Cq}}$ method normalized to GAPDH (32). To statistically compare the differences between groups, $\log _{2}$ transformation was used to normally distribute the data.

Cell co-culture and invasion. Transwell chambers with $8 \mu \mathrm{m}$ pores (cat. no. 3402; Corning, Inc.) were used for assessing cell invasion. The upper chambers were pre-coated with Matrigel (cat. no. E6909; Sigma-Aldrich; Merck KGaA). For co-culture of the HCC cells and BMECs, the 24-well Transwell co-culture system was used. HCC cells at a density of $5 \times 10^{4}$ cells and 
were plated in the upper chamber, while BMECs at a density of $2 \times 10^{5}$ cells were seeded in the lower chamber. Both chambers were supplemented with serum-free medium. Subsequently, 100 nM CX3CL1 (R\&D Systems, Inc.) or 50 ng/ml neutralizing antibody of CX3CL1 (cat. no. ab89229; Abcam) were added to the lower chamber. The cells were incubated at $37^{\circ} \mathrm{C}$ for $48 \mathrm{~h}$, and the upper surfaces were gently swabbed to remove cells which had not migrated. The cells which had migrated to the lower membrane were fixed with $1 \%$ glutaraldehyde and stained with $0.1 \%$ crystal violet (cat. no. C0775; Sigma-Aldrich) for $15 \mathrm{~min}$ at room temperature. The number of cells which had invaded were counted in 5 randomly selected fields per well. Invasion experiments were performed 3 times independently.

Cell migration. Transwell chambers with $8 \mu \mathrm{m}$ pores (cat. no. 3402; Corning, Inc.) were used for assessing cell migration. For co-culture of the HCC cells and BMECs, the 24-well Transwell co-culture system was used. HCC cells at a density of $2 \times 10^{5}$ cells and were placed in the lower chamber. While BMECs at a density of $1 \times 10^{5}$ cells were seeded in the upper chamber. Both chambers were supplemented with serum-free medium. Subsequently, 100 nM CX3CL1 (R\&D Systems) or $50 \mathrm{ng} / \mathrm{ml}$ neutralizing antibody of CX3CL1 (cat. no. ab89229; Abcam) were added to the lower chamber. Cells were grown to over the entire bottom of each well in a monolayer, a 100- $\mu 1$ pipette tip was used to scratch the monolayer, and cells were cultured in $2 \mathrm{ml}$ serum-free DMEM. Wound-healing was observed using an optical microscope after $48 \mathrm{~h}$. The experiments were repeated 3 times independently.

Cell counting kit-8 (CCK-8) assays. A total of $1 \times 10^{4}$ cells $/ \mathrm{ml}$ BMECs were seeded into a 96-well plate. Cells were incubated at $37^{\circ} \mathrm{C}$ for $24 \mathrm{~h}$, after which the media was removed and $10 \mu \mathrm{l}$ CCK-8 solution was added to each well, and further incubated at room temperature for $2 \mathrm{~h}$. The absorbance at $450 \mathrm{~nm}$ was measured using a microplate reader (Thermo Fisher Scientific, Inc.). The experiments were repeated 3 times independently.

Hematoxylin and eosin $(H \& E)$ staining. The tissues were fixed in $10 \%$ formaldehyde, dehydrated in graded ethanol, paraffin-embedded, and sliced. The tissue sections were then dewaxed in xylene, rehydrated in graded ethanol, and stained with hematoxylin for $1 \mathrm{~min}$ and eosin for $1 \mathrm{~min}$. Following dehydration in graded ethanol and vitrification in dimethylbenzene, the tissue sections were observed under an optical microscope (Olympus-IX51; Olympus Corporation). All experimental procedures were carried out at room temperature.

ELISA. Cell medium was collected and used for detecting the concentrations of CX3CL1. The levels of CX3CL1 were detected using ELISA kits (Boster Bio) in accordance with the manufacturer's instructions. The optical density (OD) at $450 \mathrm{~nm}$ was measured using a microplate reader (Bio-Rad Laboratories, Inc.).

Small interfering (si)RNA and transfection. The overexpression plasmids of ADAM17 (bank ID: NM_003183) were purchased from GeneChem, Inc. The siRNA sequences used were as follows: siCX3CL1, 5'-GGACAAGCCACATAGGAAA-3' and
siADAM17, 5'-GCUUGUUCAUCGAGUGAAAdTdT-3'. Control cells were subjected to mock transfection with scrambled sequences (GeneChem, Inc.). CX3CR1 overexpression lentivirus (bank ID: NM_001337) and CX3CL1 siRNA lentivirus (bank ID: NM_002996) were purchased from GeneChem, Inc. Cells were first seeded into 6-well plates and cultured to a cell density of $70 \%$. Subsequently, lentiviral transfection as performed according to the manufacturer's instructions. ADAM17 overexpression, siADAM17, CX3CL1 overexpression and siCX3CL1 plasmids were transfected into BMECs, respectively. CX3CR1 overexpression lentivirus was transfected into HCC cell lines. CX3CL1 siRNA lentivirus was transfected into BMECs. At 1 day prior to transfection with $100 \mathrm{nM}$ siRNA or $1 \mu \mathrm{g} / \mathrm{ml}$ plasmids, $1 \times 10^{5}$ cells/well were plated into 24 -well plates in DMEM and 10\% FBS. Lipofectamine ${ }^{\circledR} 2000$ (Invitrogen; Thermo Fisher Scientific, Inc.) was used for transfection for $6 \mathrm{~h}$ in serum-free medium at $37^{\circ} \mathrm{C}$ according to the manufacturer's protocol. Subsequently, the medium was replaced with DMEM with $10 \%$ FBS.

Animal model. A total of 40 4-6-week-old male NOD/SCID mice were obtained from Vital River Laboratory Animal Technology Co., Ltd. All animal experiments were performed in compliance with the Guidelines for the Care and Use of Research Animals established by the Animal Ethics Committee of Zhongshan Hospital, Fudan University. The animal studies were approved by the Animal Ethics Committee of Zhongshan Hospital, Fudan University. Experiments met the ethical requirements for animal experiments. $\mathrm{MHCC} 97 \mathrm{H}$ cells were infected with Luc-CX3CR1 lentivirus to establish CX3CR1-MHCC97H, a stable HCC cell line overexpressing CX3CR1. A total of 30 mice were randomly divided into 2 groups. Subsequently, $1 \times 10^{6}$ tumor cells were suspended in $200 \mu \mathrm{l}$ serum-free medium, and the cells were injected into the left ventricle of the anesthetized mice. Mice were anesthetized by an intraperitoneal injection of sodium pentobarbital (80 mg/kg). After 6-8 weeks, the mice were analyzed using in vivo imaging, micro-CT scans. The mice were sacrificed at 8 weeks by an intraperitoneal injection of sodium pentobarbital (200 mg/kg) and histological analysis was then performed. To determine whether locally administered BMECs mixed with tumor cells promoted tumor growth, $5 \times 10^{6}$ MHCC97H cells were mixed with $1 \times 10^{5}$ BMECs infected with CX3CL1-silencing lentivirus or control lentivirus and injected into the spine of the mice for primary tumor assessment. After 4 weeks, the tissues were analyzed using immunohistochemistry. The tumor long diameter (L) and short diameter (W) were measured to calculate the tumor volume. Tumor volume $(\mathrm{V})=\left(\mathrm{L} \mathrm{x} \mathrm{W}^{2}\right) / 2$ (33).

Flow cytometry. To stain for the cell surface markers, cells were incubated with following fluorescence-labeled monoclonal antibodies for $30 \mathrm{~min}$ on ice respectively: PE-conjugated anti-CD11b (cat. no. 333142; BD Biosciences; 1:300), FITC-conjugated anti-F4/80 (cat. no. orb223773; Biorbyt; 1:300), PE-Cy7-conjugated-CD45 (cat. no. 147703; BioLegend; 1:300), APC-conjugated CX3CR1 (cat. no. 341609; BioLegend; 1:300), PE-Cy3-conjugated MRC1 (cat. no. sc-376232; Santa Cruz Biotechnology, Inc.; 1:300) and PE-Cy5-CD163 (cat. no. sc-33715; Santa Cruz Biotechnology, Inc.; 1:300) antibodies. Macrophages population in peripheral 

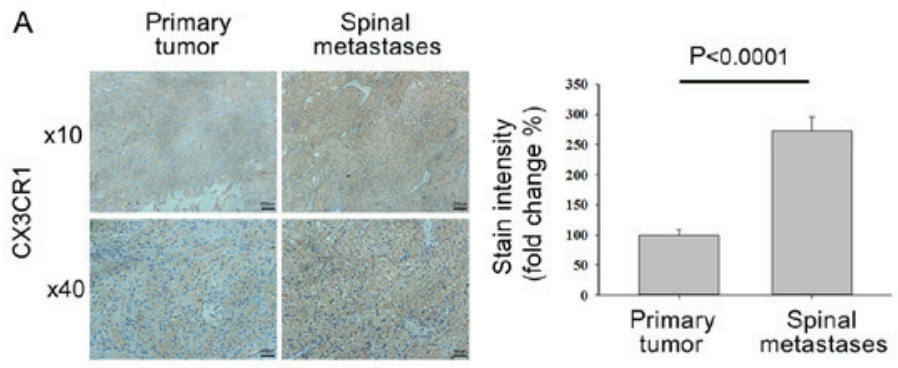

C

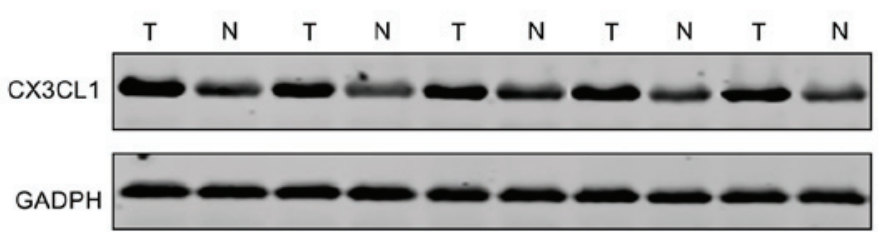

B

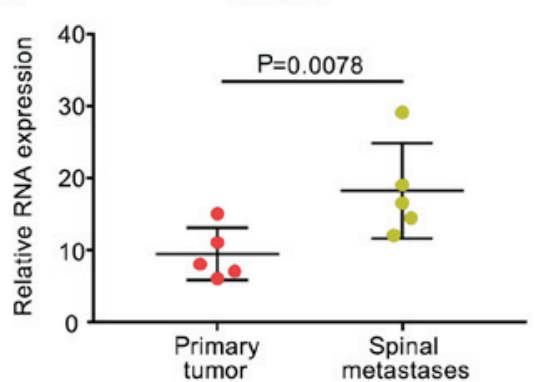

D

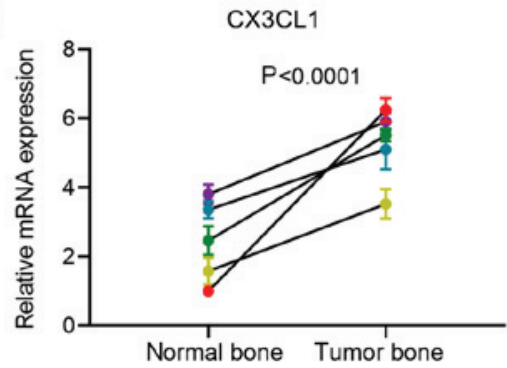

Figure 1. Expression of CX3CL1/CX3CR1 is upregulated in spinal metastases. (A) Immunohistochemistry was used to compare differences in the expression of CX3CR1 between liver spinal metastases tissues and primary liver cancer. Scale bar, $200 \mu \mathrm{m}$. (B) mRNA expression levels of CX3CR1 in liver spinal metastases tissue and primary HCC. $\mathrm{n}=5$ per group. (C) Western blot analysis of protein expression of CX3CL1 in tumor bone and normal vertebral bone; $\mathrm{n}=5$ per group. (D) mRNA expression levels of $C X 3 C L 1$ in liver spinal metastases tissue and primary HCC; $\mathrm{n}=3$ per group. Data were analyzed using a paired Student's t-test following log transformation. N, normal vertebral bone; T, tumor bone; HCC, hepatocellular carcinoma; CX3CL1, C-X3-C motif chemokine ligand 1; CX3CR1, C-X3-C motif chemokine receptor 1.

blood of mice was determined based on the co-expression of CD45, CD11b and F4/80. Cells were selected based on the higher expression of CD45 for macrophages. CD11b and F4/80 expression were used for the further characterization of the macrophages. Data were acquired using a fluorescence activated cell sorting (FACS)Calibur (BD Biosciences), and analyzed using CellQuest software (BD Biosciences).

Statistical analysis. The staining intensity of immunohistochemical and fluorescence the intensity of immunofluorescent were measured through integrated optical density/area in Image-Pro Plus 6.0. SPSS version 16.0 (SPSS, Inc.) was used to analyze all data. Data are presented as the means \pm standard deviation of 3 experimental repeats. The $\chi^{2}$ test was used to analyze the in vivo experiments (spinal metastasis rate). Otherwise, ANOVA with a post hoc Tukey's test, a Student's t-test, Mann-Whitney U or Kruskal Wallis test followed by Dunn's non-parametric post hoc test were used to compare differences between groups. $\mathrm{P}<0.05$ was considered to indicate a statistically significant difference. The Mann-Whitney $U$ test is a non-parametric test of the null hypothesis that two samples are derived from the same population against an alternative hypothesis, prinicipally that a particular population tends to have larger values than the other. Unlike the t-test, it does not require the assumption of normal distributions. It is almost as efficient as the t-test on normal distributions (34).

\section{Results}

CX3CL1 and CX3CR1 expression are upregulated in spinal metastases. To investigate the expression of CX3CR1 and CX3CL1 in primary and metastatic tumors, tissue sections and total protein samples were isolated during surgery from patients with or without HCC spinal metastases. Immunohistochemistry revealed that the CX3CR1 levels in HCC spinal metastases were upregulated compared with the primary tumors (Fig. 1A). The mRNA expression levels of CX3CR 1 were also higher in HCC spinal metastases compared with primary tumors (Fig. 1B). CX3CL1 expression in healthy vertebral bone was compared with that of bone tumors from patients with HCC. Western blot analysis revealed that the protein expression levels of CX3CL1 were higher in tumor bone compared with healthy vertebral bone (Fig. 1C). Consistent with the increase in protein levels, the mRNA expression levels of $C X 3 C L 1$ was also increased in tumor bone compared with healthy vertebral bone (Fig. 1D). These differences in expression support the hypothesis that CX3CR1 and CX3CL1 serve a critical role in spinal metastases.

CX3CL1 promotes HCC cell invasion and migration through the Src signaling pathway. A previous study demonstrated that CX3CL1 was not only significantly expressed in spinal cancellous bone, but also in the metastatic bone of patients with HCC (26). As a chemokine, CX3CL1 has been reported to enhance increased cancer cell invasion and migration (35). In order to investigate whether CX3CL1 binds to its receptor, CX3CR1, to enhance the migration of HCC cells, migration assays were performed using Transwell chambers. CX3CL1 significantly increased the migration of HCC cells compared with the control, and higher concentrations of CX3CL1 further enhanced migration (Fig. 2A). Subsequently, invasion assays were performed. The results confirmed that CX3CL1 significantly increased the invasion of $\mathrm{MHCC} 97 \mathrm{H}$ cells compared with the control (Fig. 2B). 
A
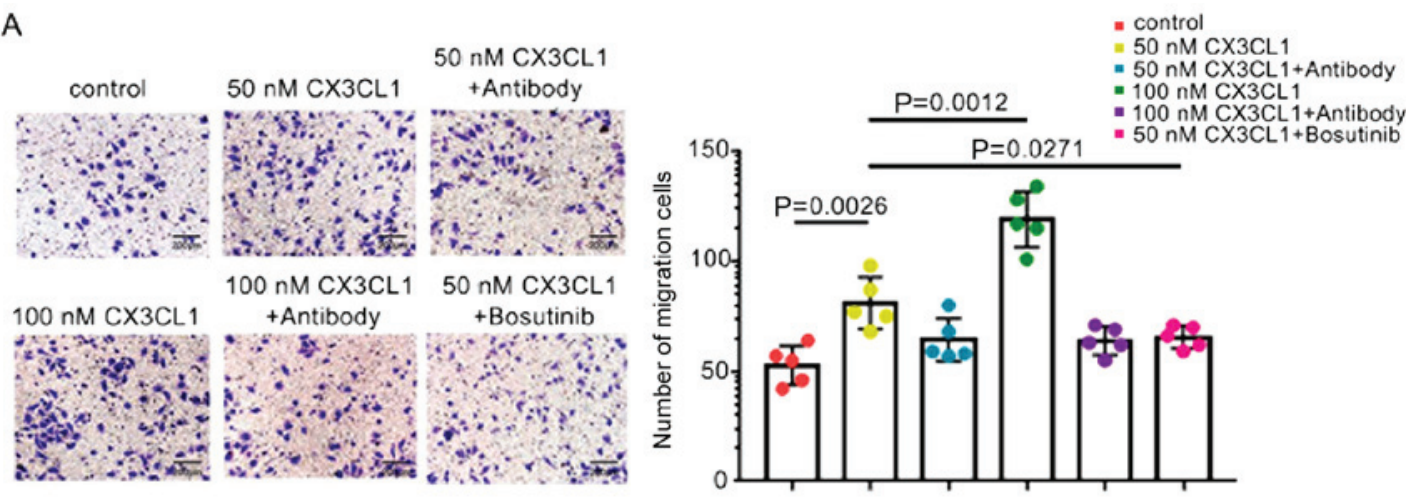

B
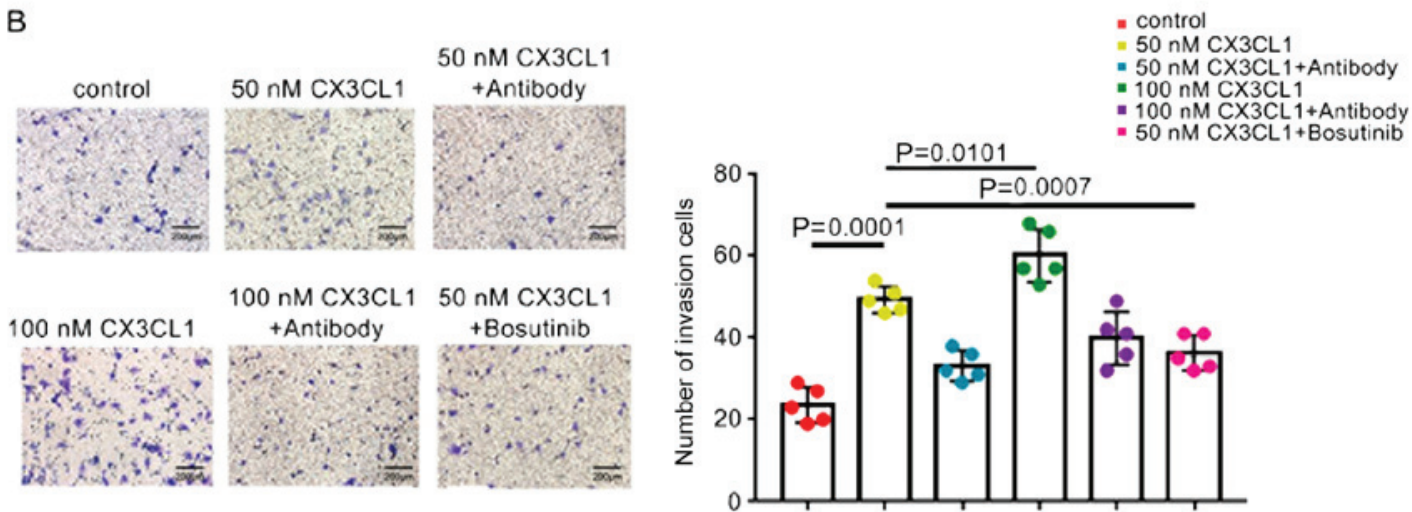

$\mathrm{C}$
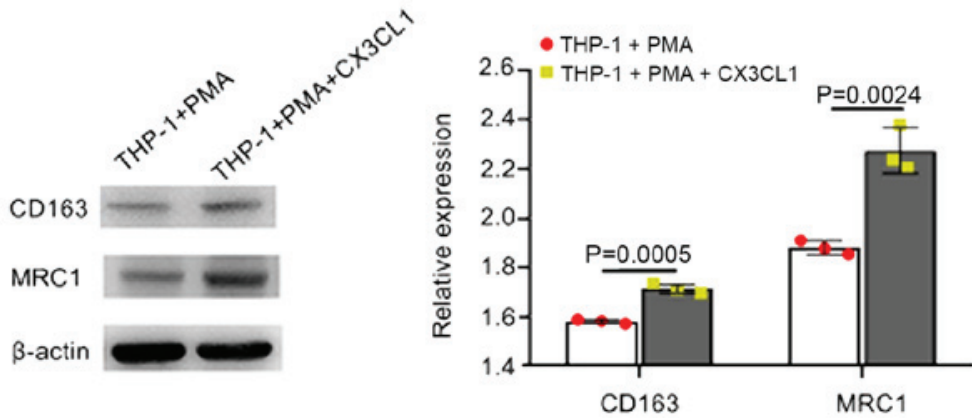

Figure 2. CX3CL1 promotes the invasion and migration of HCC cells through Src. A total of 50 or $100 \mathrm{nM}$ recombinant human CX3CL1 was added to the medium. CX3CL1 antibody was added to neutralize free CX3CL1 in medium. Bosutinib was used to inhibit Src. (A) Representative images and statistical analysis of Transwell migration assays showed the migratory ability of HCC cells. The number of cells which had invaded was quantified; n=5; scale bar, $200 \mu \mathrm{m}$. (B) Representative images and bar graphs of Transwell invasion assays showed the invasive ability of HCC cells after $24 \mathrm{~h}$. The number of cells which had migrated was quantified; $\mathrm{n}=5$; scale bar, $200 \mu \mathrm{m}$. Cell migration and invasion were analyzed using ANOVA. (C) Expression of THP- 1 cells surface markers $\mathrm{MRC1}$ and $\mathrm{CD} 163$ were detected by western blot analysis; $\mathrm{n}=3$. Western blot analysis data were analyzed using a Student's $\mathrm{t}$-test following log transformation. HCC, hepatocellular carcinoma; CX3CL1, C-X3-C motif chemokine ligand 1; MRC1, mannose receptor C-type 1.

Through high-throughput analysis in our previous studies, it was shown that the Src/PTK2 pathway may participate in regulating CX3CL1-enhanced cell migration and invasion (27). Initially, the role of Src in cell invasion and migration was determined. The Src protein inhibitor, bosutinib, suppressed the migration and invasion of HCC cells (Fig. 2A and B). These results suggest CX3CL1 may enhance the migration and invasion of $\mathrm{HCC}$ via Src.

CX3CL1/CX3CR1 has also been shown to promote macrophages to exhibit M2 phenotypes (36). In addition, tumor-associated macrophages (TAMs) also exhibit an M2 phenotype during tumor development. Therefore, in the present study, the effect of CX3CL1 on macrophages was determined. THP-1 cells were induced to differentiate into resting macrophages using PMA. CX3CL1 was subsequently added to the
PMA-induced THP-1 cells for $48 \mathrm{~h}$. The expression levels of the M2 markers, MRC1 and CD163, were examined by western blot analysis and was found to be significantly increased compared with the controls (Fig. 2D). These results suggest that TAMs differentiate into the M2 morphological phenotype following CX3CL1 stimulation.

CX3CL1 facilitates HCC spinal metastasis in a mouse model. To examine the effects of CX3CL1 in HCC spinal metastases, a nude mouse model of HCC metastasis was established by injecting $\mathrm{HCC}$ cells directly into the left ventricle of nude mice. Lentiviral transfection was used to overexpress CX3CR1 in MHCC97H cells and establish a CX3CR1-MHCC97H cell line (Fig. S1A). After 6 weeks, the mice were examined by performing in vivo imaging to investigate whether spinal 
A

Rate of mouse HCC spinal metastasis

\begin{tabular}{lccc}
\hline & control & CX3CR $1^{\text {high }}$ group & P-value \\
\hline Spinal metastasis & 2 & 7 & 0.0464 \\
Without spinal metastasis & 13 & 8 & \\
Metastasis rates & $13.33 \%$ & $46.67 \%$ & \\
\hline
\end{tabular}

B

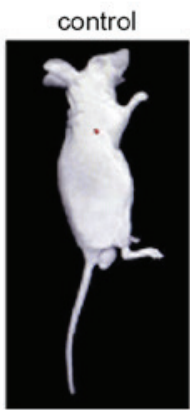

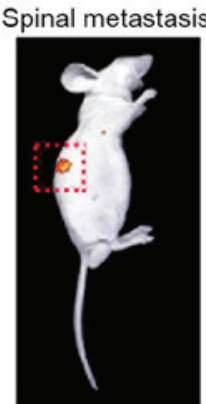

C

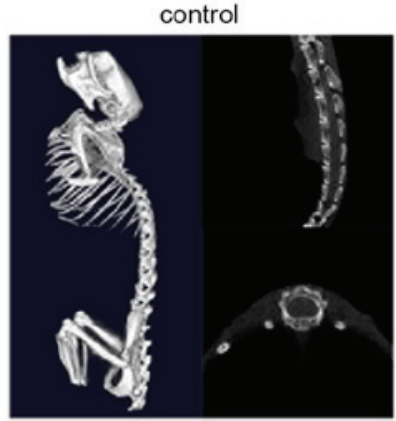

Spinal metastasis

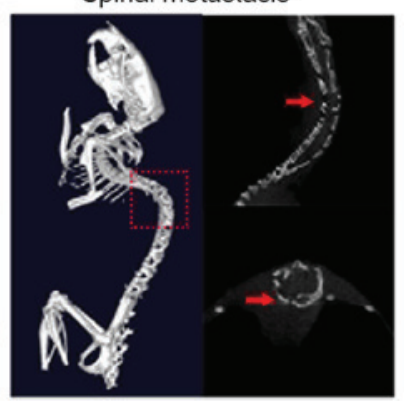

D

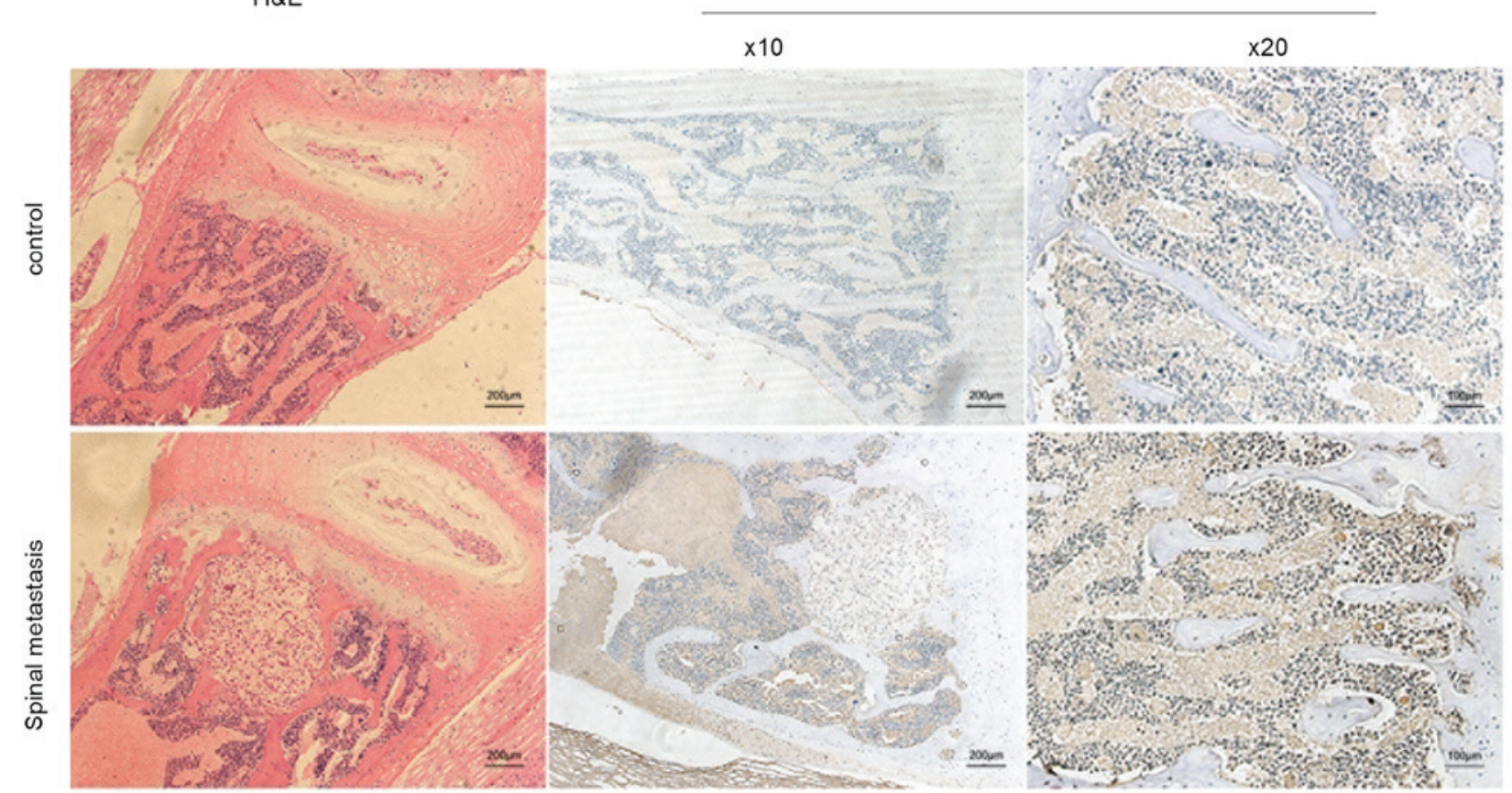

Figure 3. CX3CL1 facilitates HCC spinal metastasis in a mouse model. A total of 1x106 MHCC97H or CX3CR1-MHCC97H cells were resuspended in $200 \mu 1$ serum-free medium and injected into the left ventricles of mice. (A) Statistical results of spinal metastasis. Data were analyzed using a $\chi^{2}$ test. (B) In vivo imaging of metastasis in the mice model. Red dotted box showed the distant metastases. (C) Lesions of spinal metastasis underwent a further micro-CT scan. Micro-CT scans illustrated local destruction (arrow) in the affected vertebrae of the tumorigenic mice. (D) Hematoxylin and eosin staining and immunohistochemical staining of CX3CL1 in the paracancerous tissues and healthy spine tissues. Scale bar, $200 \mu \mathrm{m}$. HCC, hepatocellular carcinoma; CX3CL1, C-X3-C motif chemokine ligand 1 .

metastasis had occurred. In total, 2 spinal metastases were visualized in the control group; however, 7 spinal metastases were visualized in the CX3CR1-MHCC97H group (Fig. 3A and B). In addition, Micro-CT was also used to further analyze the spinal metastases by inspecting for bone damage (Fig. 3C). The results indicated that spinal metastasis was enhanced in nude mice injected with CX3CR1-MHCC97H cells compared with the controls (Fig. 3A). Subsequently, CX3CL1 levels in spinal cancellous bone from the control and CX3CR1-MHCC97H groups were compared. The cancellous bone collected from the mice with CX3CR1-expressing tumor cells exhibited higher CX3CL1 levels compared with the controls (Fig. 3D).
These results suggest that HCC spinal metastasis is increased in spinal cancellous bone expressing higher levels of CX3CL1.

BMECs promote the invasion and migration of HCC cells through CX3CL1. BMECs are responsible for forming the hematopoietic microenvironment and modulating hematopoietic cells; thus, they exhibit a relatively high ability to secrete cytokines. BMECs are a major source of cytokines in the bone marrow microenvironment, and a previous study reported that BMECs produce CX3CL1 (37). To elucidate the specific regulatory mechanisms used by BMECs on CX3CL1, the CX3CL1 levels in BMECs were compared with HUVECs. Primary 
A
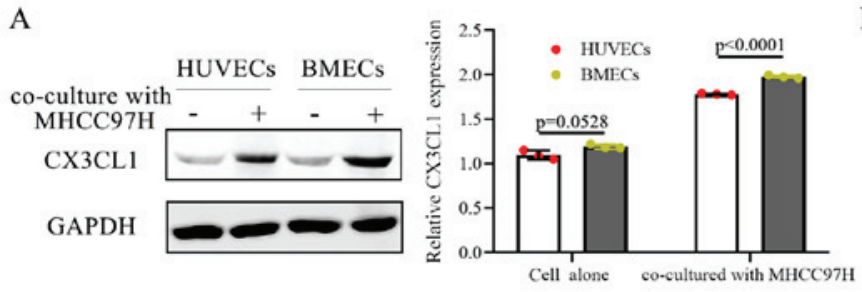

$\mathrm{C}$

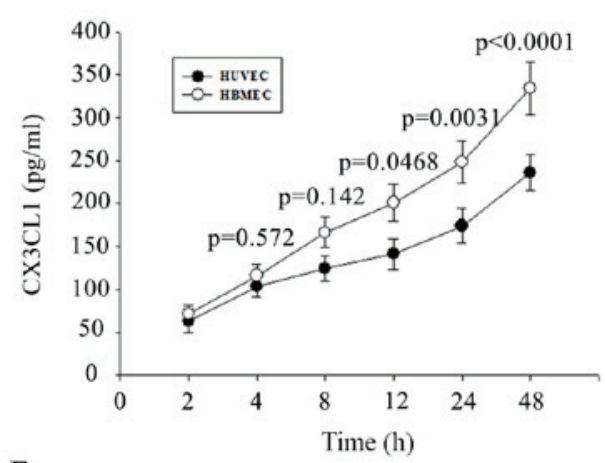

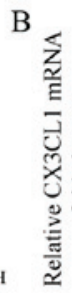

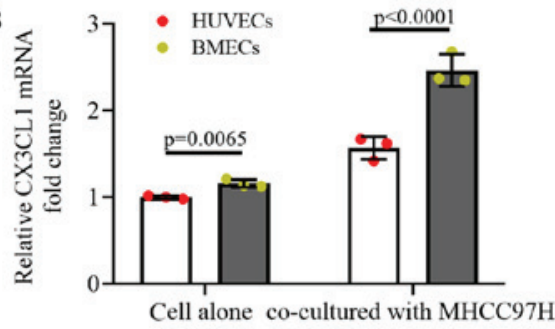

E
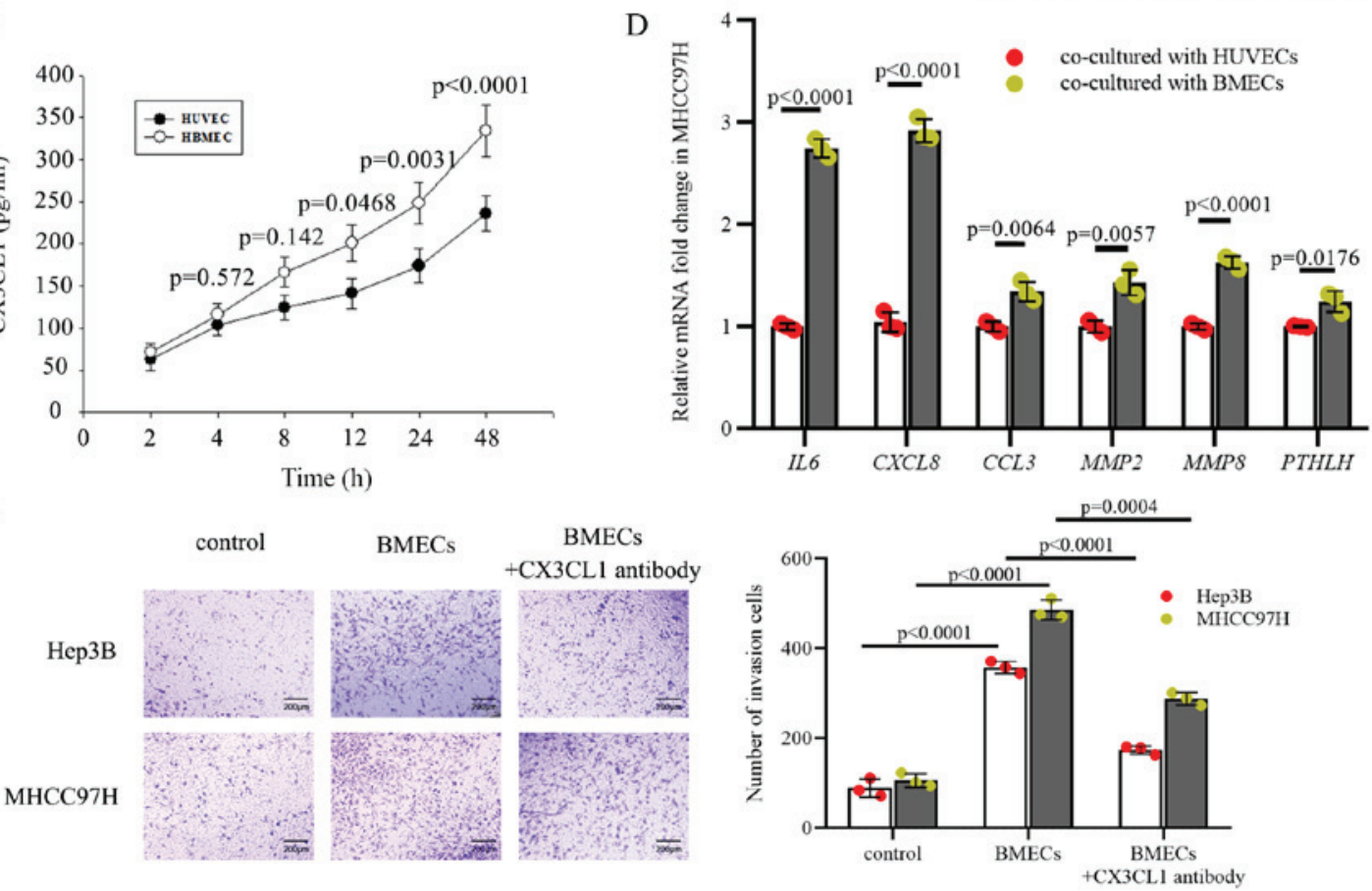

$\mathrm{F}$
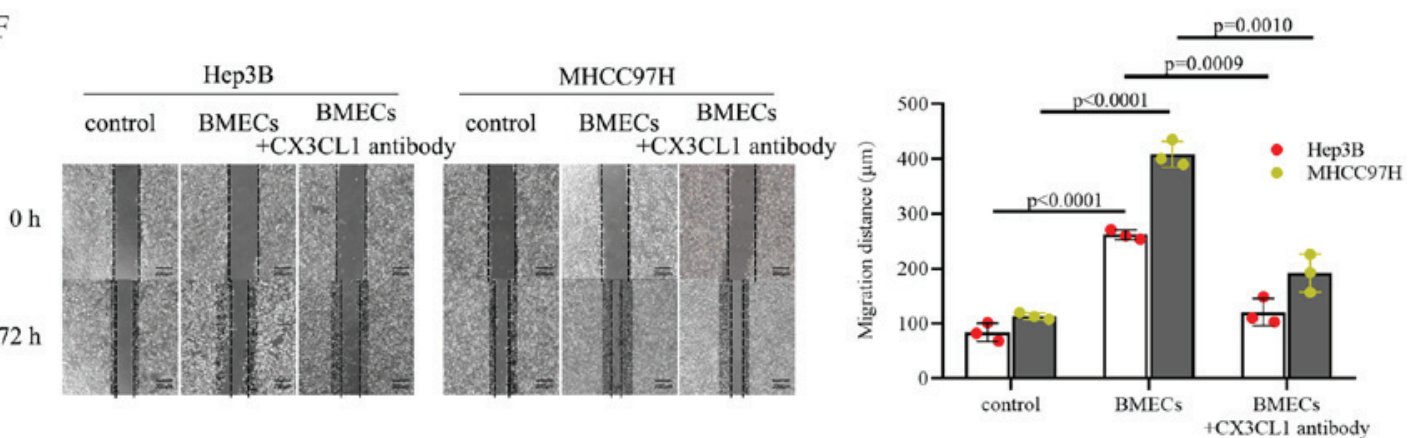

Figure 4. BMECs promote the invasion and migration of HCC cells through CX3CL1. (A) Protein and (B) mRNA expression levels of CX3CL1 in HUVECs and BMECs cells were examined. RT-qPCR and western blot analysis data were analyzed using a Student's t-test following log transformation. (C) ELISA was used to examine secreted CX3CL1 expression in BMECs co-cultured with MHCC97H cells. (D) mRNA expression levels of CXCL8, IL6, CCL3, MMP2, $M M P 8$ and $P T H L H$ in MHCC97H cells. n=3. Hep3B and MHCC97H cells were co-cultured with BMECs in Transwell chambers. CX3CL1 antibody was used to neutralize the free CX3CL1 in medium. Western-blot and PCR data were analyzed using a Student's t-test following log transformation. (E) Representative images and statistical analysis of Transwell invasion assays showed the invasive ability of Hep3B and MHCC97H cells co-cultured with or without BMECs after $24 \mathrm{~h} ; \mathrm{n}=3$. The number of the cells which had invaded was quantified. Scale bar, $200 \mu \mathrm{m}$. (F) Representative images and analysis of wound healing assays showed the migratory ability of Hep3B and MHCC97H cells co-cultured with or without BMECs after $24 \mathrm{~h}$. Migration distance of cells was quantified; $\mathrm{n}=3$; scale bar, $200 \mu \mathrm{m}$. Cell migration and invasion were analyzed using ANOVA test; $\mathrm{n}=3$. BMECs, bone marrow epithelial cells; HCC, hepatocellular carcinoma; CX3CL1, C-X3-C motif chemokine ligand 1.

BMECs were isolated from human bone marrow samples and it was shown that the CX3CL1 protein and mRNA levels were upregulated in BMECs co-cultured with MHCC97H cells and were higher compared with HUVECs (Fig. 4A and B). Given that CX3CL1 is a secreted chemokine and can promote chemotaxis, the CX3CL1 protein levels in media conditioned by BMECs were measured using an ELISA kit. The results indicated that BMECs released increased quantities of CX3CL1 into the extracellular environment compared with HUVECs (Fig. 4C). Based on the above results, it was hypothesized that BMECs serve as a crucial source of CX3CL1. Bone destruction is one of the crucial steps required for tumor metastasis. MHCC $97 \mathrm{H}$ cells co-cultured with BMECs displayed higher mRNA expression levels of $C X C L 8$, interleukin (IL)6, CCL3, MMP2, MMP8 and parathyroid hormone like hormone (PTHLH) (Fig. 4D), all of which are strictly associated with bone destruction in 
A
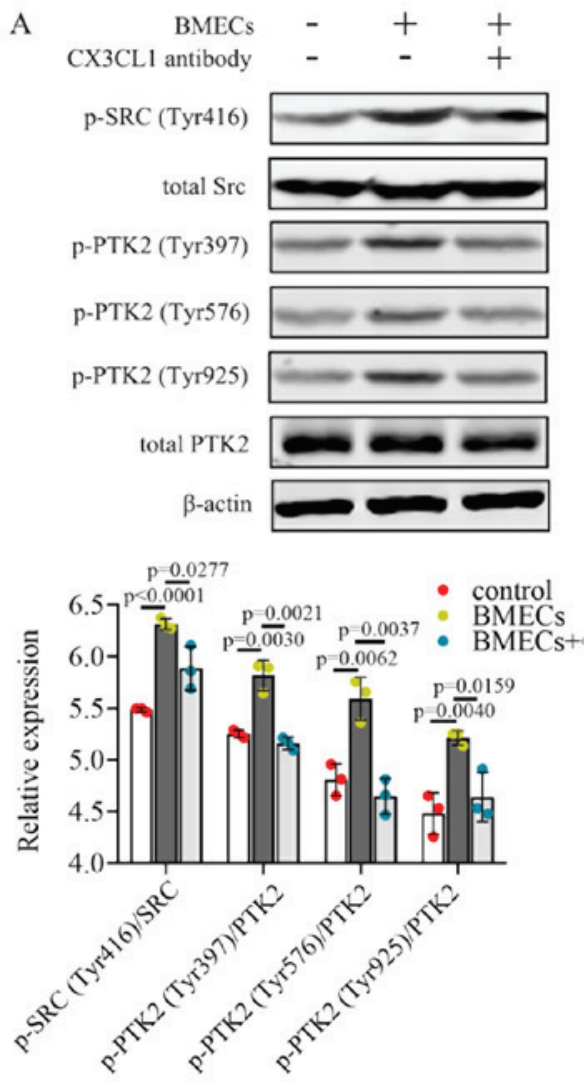

B
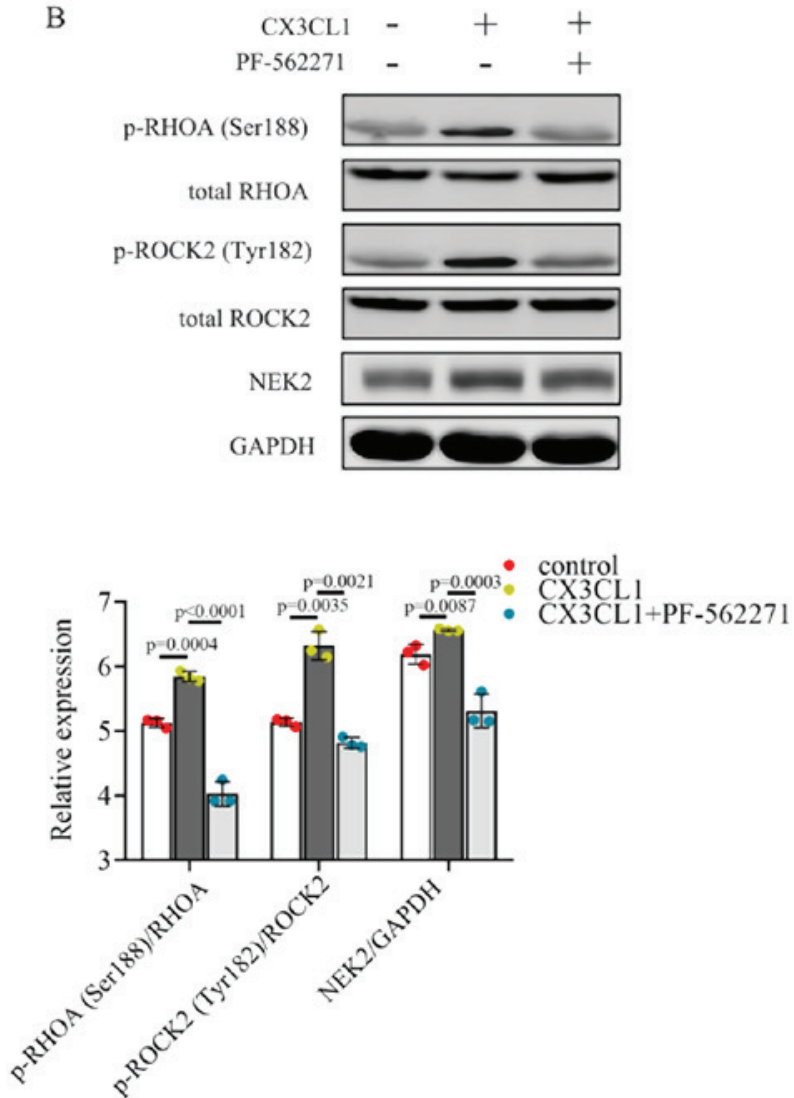
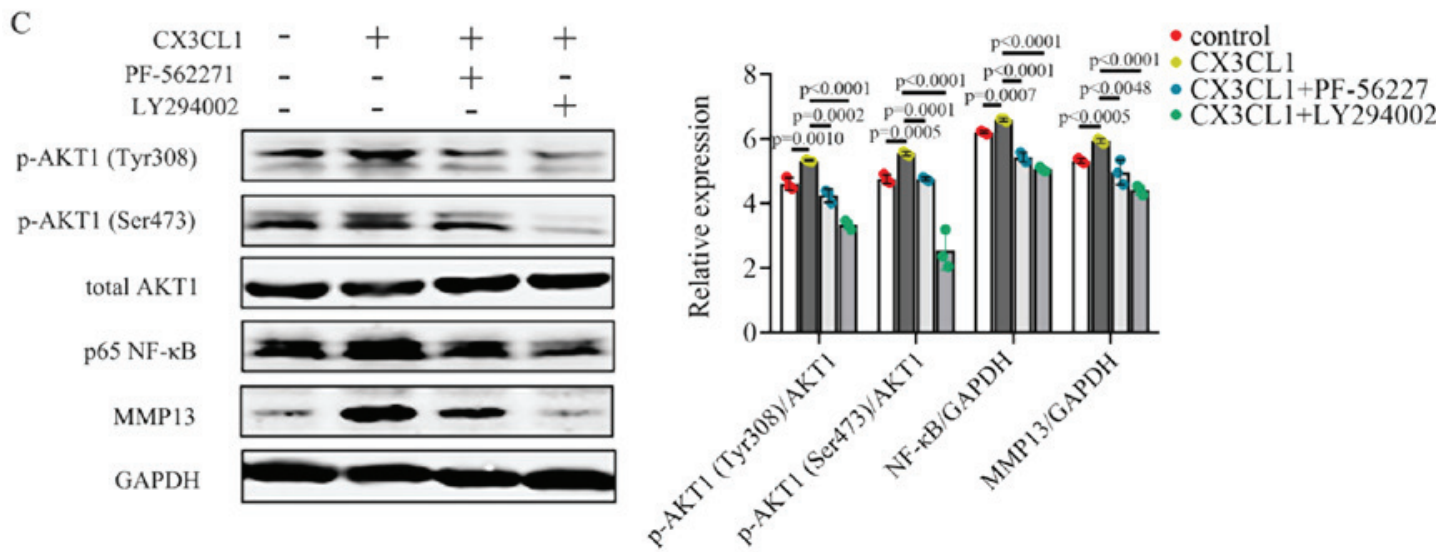

Figure 5. CX3CL1 increases the activation of the PIK3CA/AKT1 and RHOA/ROCK2 signaling pathways. (A) Protein expression levels of p-Src (Tyr416), total Src, p-PTK2 (Tyr397/Tyr576/Tyr925), total PTK2 and $\beta$-actin were assessed using western blot analysis;. n=3. (B) p-RHOA (Ser188), total RHOA, p-ROCK2 (Tyr182), total ROCK2, NEK2 and GAPDH were measured by western blot analysis; n=3. (C) p-AKT1 (Tyr308/Ser473), total AKT1, p65 NF-кB and MMP13 were detected by western blot analysis; $\mathrm{n}=3$. western blot data were analyzed using ANOVA and Tukey's test. p-, phosphor; HCC, hepatocellular carcinoma; CX3CL1, C-X3-C motif chemokine ligand 1.

cancer (38). Taken together, these results suggest that BMECs express and secrete CX3CL1 into the bone marrow to attract metastatic cells into the vertebral body and to regulate the surrounding tumor microenvironment.

To determine whether BMEC-derived CX3CL1 directly regulates the function of tumor cells, cell invasion was measured using Transwell assays and migration was measured using wound-healing assays. The presence of BMECs increased the invasion and migration of $\mathrm{MHCC} 97 \mathrm{H}$ and $\mathrm{Hep} 3 \mathrm{~B}$ cells (Fig. 4E and F). When CX3CL1 in BMEC-conditioned media was neutralized using an CX3CL1 antibody, the invasion and migration activity of the MHCC97H and Hep3B cells was significantly decreased (Fig. 4E and F), suggesting that
BMECs release soluble CX3CL1 to facilitate the invasion and migration of HCC cells.

CX3CL1 increases the activation of the PIK3CA/AKT1 and RHOA/ROCK2 signaling pathways. BMECs promoted the activation of p-PTK2 (Tyr397/Tyr576/Tyr925), as shown by the increase in $\mathrm{p}$-Src (Tyr416) protein expression in MHCC97H cells (Fig. 5A). Additionally, a reduction in p-Src (Tyr416) and p-PTK2 (Tyr397/Tyr576/Tyr925) protein levels was also observed when CX3CL1 was neutralized (Fig. 5A). These results suggest that BMECs release CX3CL1 which upregulates the activation of Src/PTK 2 signaling in MHCC97H cells. 
A BMECs co-cultured with

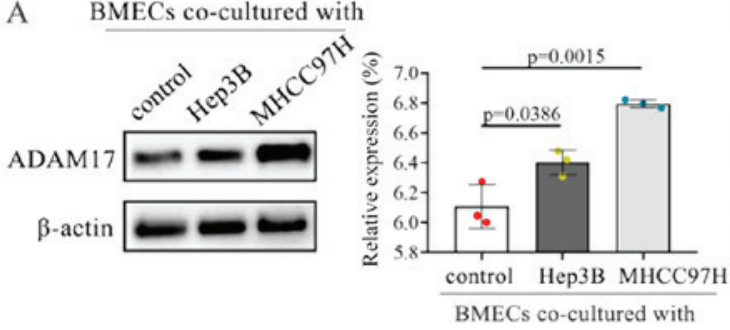

D

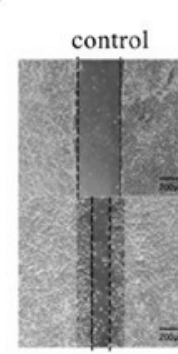

I

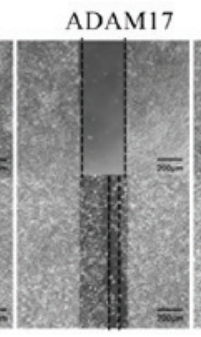

II

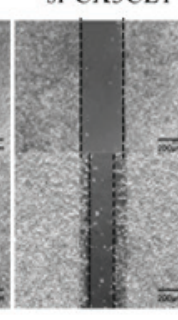

III

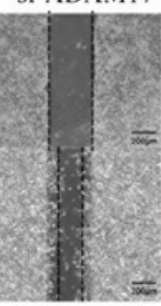

IV
B

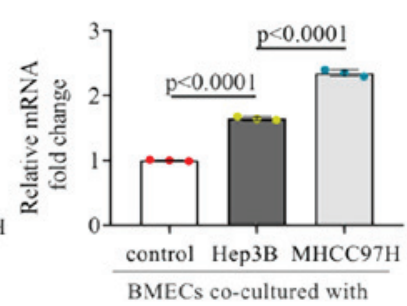

C g control

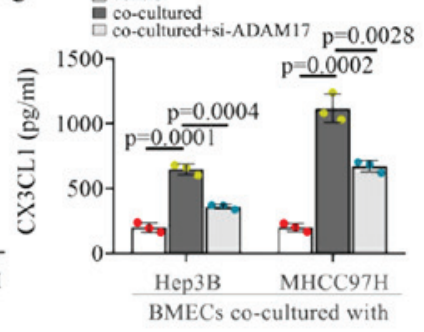

E

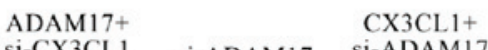
si-CX3CL1 $\quad$ si-ADAM17 si-ADAM17

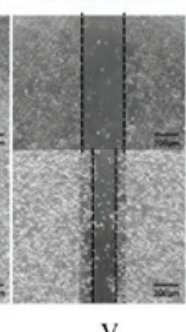

$\mathrm{V}$

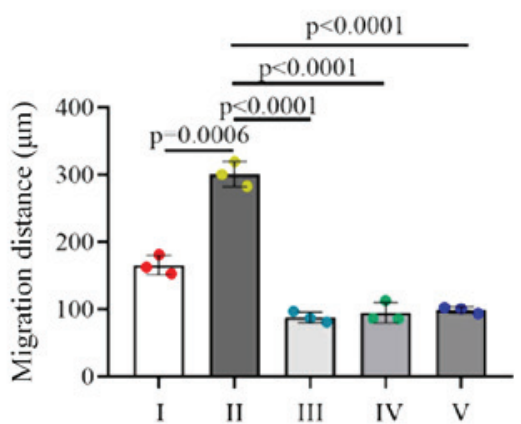

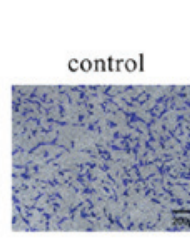

I

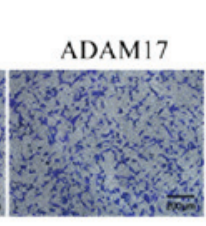

II co-cultured with

F

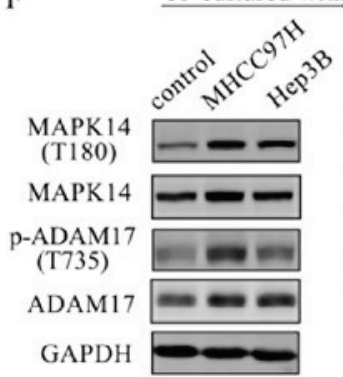

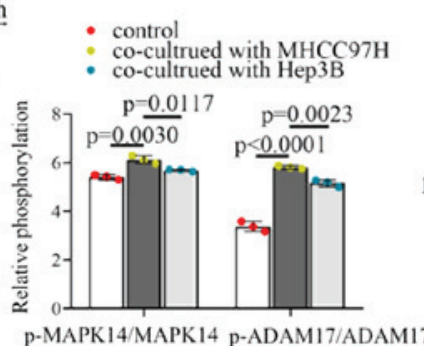
p-MAPK14/MAPK14 p-ADAM17/ADAMI7

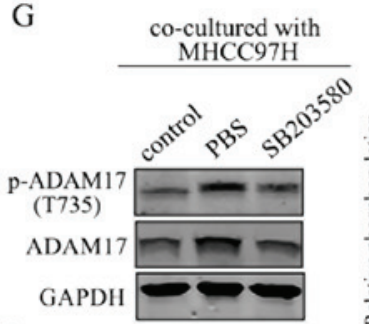

GAPDH
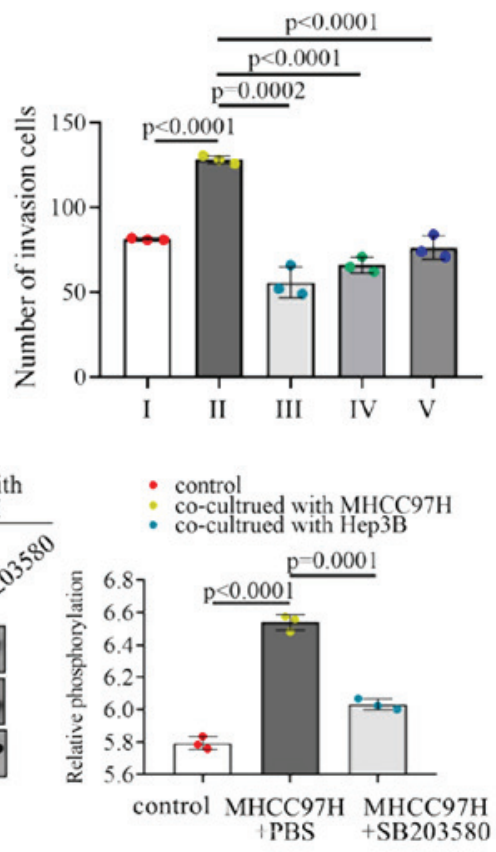

Figure 6. ADAM17 in BMECs is activated by MAPK14 promoted invasion and migration of HCC cells by regulating CX3CL1. siRNA or overexpression plasmids were transfected into BMECs. (A) Protein levels of ADAM17 in BMECs co-cultured with Hep3B or MHCC97H were examined using western blot analysis; $\mathrm{n}=3$. (B) mRNA levels of ADAM17 in BMECs co-cultured with Hep3B or MHCC97H; $\mathrm{n}=3$. (C) si-ADAM17 was transfected into BMECs to reduce ADAM17 expression. ELISA was used to examine the expression of CX3CL1 secreted by BMECs into the medium when co-cultured with Hep3B or MHCC97H cells; $n=3$. (D) Representative images and statistical analysis of Transwell invasion assays showed the invasive ability of MHCC $97 \mathrm{H}$ cells co-cultured with BMECs after $24 \mathrm{~h}$. The number of the invaded cells was quantified; $\mathrm{n}=3$. Scale bar, $200 \mu \mathrm{m}$. (E) Representative images and analysis of wound healing showed the migratory ability of MHCC $97 \mathrm{H}$ cells co-cultured with BMECs after $24 \mathrm{~h}$. Migration distance of cells was quantified. $\mathrm{n}=3$; scale bar, $200 \mu \mathrm{m}$. (F) Western blot analysis was used to examine phosphorylation of ADAM17 and MAPK14 in BMECs co-cultured with Hep3B or MHCC97H; n=3. (G) SB203580 was used to inhibit MAPK14 activation. Western blot analysis was used to examine phosphorylation of ADAM17; $\mathrm{n}=3$. Western blot analysis, RT-qPCR and ELISA data were analyzed using the ANOVA and Tukey's test. Cell migration and invasion were analyzed using ANOVA test. ADAM17, a disintegrin and metalloproteinase; HCC, hepatocellular carcinoma; BMECs, bone marrow epithelial cells; si, small interfering; CX3CL1, C-X3-C motif chemokine ligand 1.

PIK3CA/AKT1 and RHOA/ROCK2 are both crucial for cell invasion and migration. The PI3K/AKT signaling pathway plays an important role in cancer for the regulation of cell growth and survival, which have long been well described (39). RHOA/ROCK 2 has been shown to be linked to the contraction and elongation of the cytoskeleton which is closely associated with cancer metastasis (40). These two pathways have been confirmed to be involved in HCC progression $(41,42)$. To verify the effects of PTK2 on CX3CL1-induced MHCC97H cells, cells were treated with the PTK2 inhibitor, PF562271. Exposure to CX3CL1 resulted in the induction of p-RHOA (Ser188)/p-ROCK2 (Tyr182) and increased NEK2 expression levels (Fig. 5B). PF562271 treatment reversed activation of the RHOA/ROCK2/NEK2 signaling pathway (Fig. 5B). NEK2 facilitates the migration and invasion of $\mathrm{HCC}$, and higher NEK2 levels are associated with a less favorable 
A

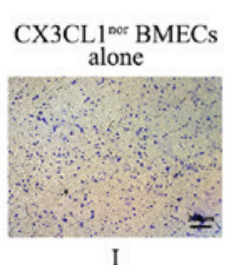

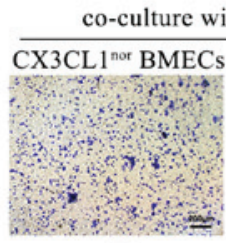

II

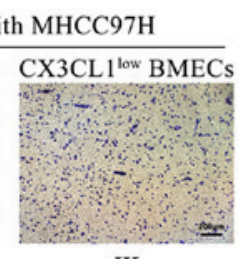

III

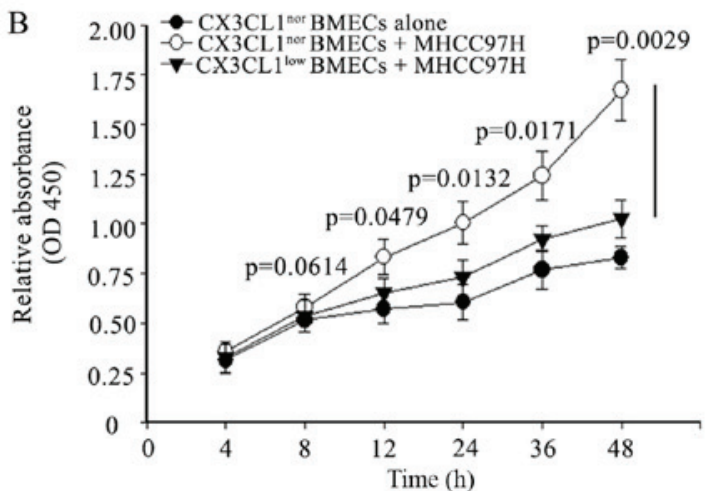

D

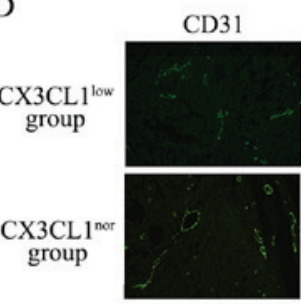

p-ADAM17
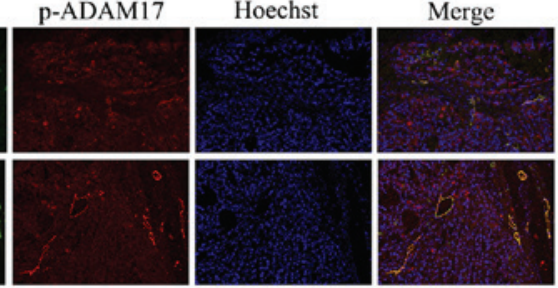

E
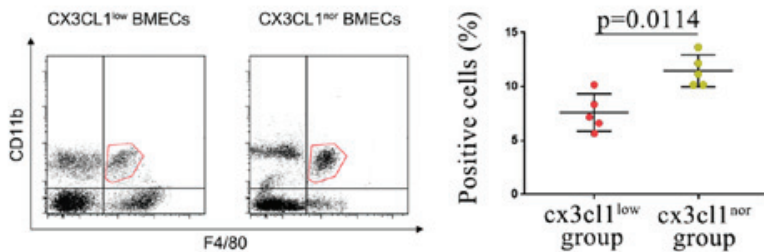

$\mathrm{F}$

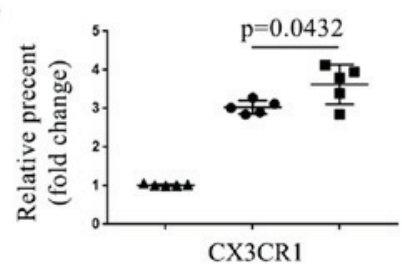

$\Delta$ Monocyte

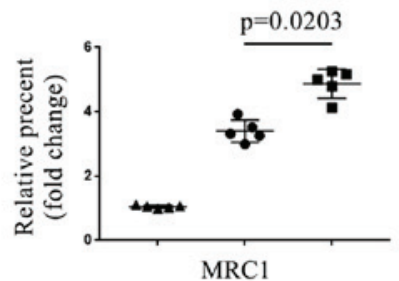

$\mathrm{cx} 3 \mathrm{cl} 1^{\text {nor }}$ group
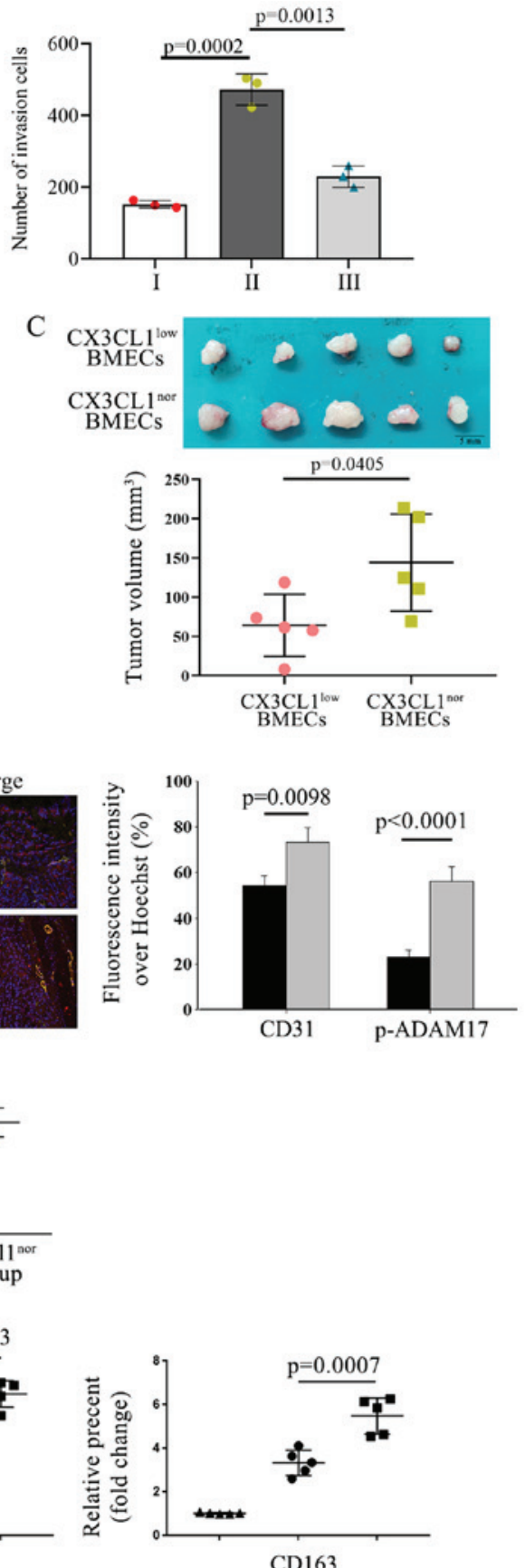

CD163

Figure 7. Interaction between BMECs and HCC cells enhances tumor growth in the spine. (A) si-CX3CL1 was transfected into BMECs to construct CX3CL1 ${ }^{\text {low }}$ BMECs. Transwell assays were used to examine recruitment of BMECs co-cultured with or without HMCC97H cells. The number of the recruited cells was quantified. $n=3$. Cell recruitment were analyzed using ANOVA test. (B) Cell vitality of BMECs was assessed using CCK- 8 assay; $n=3$. Data were analyzed using ANOVA followed by Tukey's test. (C) Tumor size was measured based on the volume. $\mathrm{n}=5$. Scale bar, $5 \mathrm{~mm}$. Data were analyzed using an unpaired t-test. (D) Samples from 2 groups were examined using immunofluorescence. Magnification, x20. The result was analyzed using an unpaired t-test. Gray bars represent the CX3CL1 $1^{\text {nor }}$ BMECs group and black bars represent the CX3CL1 $1^{\text {low }}$ BMECs group (E) Flow cytometry revealed the percentage and number of $\mathrm{F} 4 / 80^{+} \mathrm{CD} 11 \mathrm{~b}^{+}$ cells; $n=5$. (F) Flow cytometry was used for detecting expression of M2 macrophage surface markers, CX3CR1, MRC1 and CD163; n=5. Cells were selected based on higher expression of CD45 for macrophages. Subsequently, CD11b and F4/80 expression were used for further characterization of macrophages. FACS (fluorescent-activated cell sorting) data were analyzed using a Kruskal Wallis test followed by Dunn's non-parametric post hoc test. HCC, hepatocellular carcinoma; BMECs, bone marrow endothelial cells; si, small interfering; CCK-8, Cell Counting kit-8; CX3CL1, C-X3-C motif chemokine ligand 1.

prognosis of patients with HCC $(43,44)$. Both the PTK2 inhibitor, PF562271, and the PIK3CA inhibitor, LY294002, downregulated the CX3CL1-induced activation of p-AKT1 (Tyr308/Ser473) (Fig. 5C). The expression levels of both $\mathrm{NF}-\mathrm{kB}$ and its downstream effector, MMP13, were decreased when the cells were treated with PF562271 (Fig. 5C). Taken together, these data illustrate that CX3CL1 induces the activation of the RHOA/ROCK 2 and PIK3CA/AKT1 signaling pathways in HCC cells.

MAPK14 activation of ADAM17 in BMECs promotes the invasion and migration of $\mathrm{HCC}$ cells by regulating CX3CL1. 
Upon arriving at the metastatic site, tumor cells can alter the expression of genes in endothelial cells, thereby promoting tumor progression (45). Thus, whether HCC cells can influence BMECs to alter related genes in the process of CX3CL1 production was assessed in the present study. It was hypothesized that adjacent HCC cells may impact the regulation of normal BMECs in the bone marrow microenvironment. CX3CL1 has a mucin-like domain which contains a cleavage site that allows metalloproteases, such as ADAM17, to cleave and release a soluble form of CX3CL1 (46). Both $\mathrm{MHCC} 97 \mathrm{H}$ and Hep3B cells promoted the expression of ADAM17 in BMECs (Fig. 6A). The mRNA levels of ADAM17 were also upregulated when the BMECs were co-cultured with MHCC97H or Hep3B cells (Fig. 6B). Furthermore, the co-culture of BMECs with MHCC97H and Hep3B cells resulted in increased levels of soluble CX3CL1 compared with the controls (Fig. 6C). Inhibition of ADAM17 in BMECs decreased the CX3CL1 secretion (Fig. 6C). Above-mentioned results suggest that ADAM17 may effectively promote the secretion of soluble CX3CL1 from BMECs.

The expression levels of CX3CL1 were inhibited by transfection with CX3CL1 siRNA, or were promoted by transfection with CX3CL1 overexpression plasmid (Fig. S1B). ADAM17 overexpression or si-ADAM17 was also transfected into BMECs to regulate the overexpression of ADAM17 (Fig. S1C). The knockdown of ADAM17 inhibited CX3CL1 secretion from BMECs. Thus, whether the knockdown of ADAM17 reversed the CX3CL1-induced promotion of cell invasion and migration was examined. The knockdown of ADAM17 in BMECs resulted in a downregulation of the invasion and migration of MHCC97H cells, whereas ADAM17 overexpression increased cell proliferation and migration (Fig. 6D and E). Furthermore, treatment with recombinant CX3CL1 enhanced the invasion and migration of HMCC97H, whereas inhibition of ADAM17 attenuated this enhancement (Fig. 6D and E). These results suggest that the knockdown of ADAM17 significantly blocked the CX3CL1-induced invasion and migration of HCC cells.

It has been reported that the activation of ADAM17 is dependent on phosphokinases, such as MAPK14 (47). Therefore, the role of MAPK14 in the tumor cell-mediated activation of ADAM17 was assessed. The co-culture of BMECs with MHCC $97 \mathrm{H}$ or Hep3B cells induced the activation of MAPK14 and the phosphorylation of ADAM17 (Fig. 6F). The MAPK14 inhibitor, SB203580, inhibited the phosphorylation of ADAM17 at residue T735 (Fig. 6G). Thus, MAPK14 activation is crucial for the activation of ADAM17 in BMECs co-cultured with HCC cells.

Interaction between BMECs and HCC cells enhances tumor growth in the spine. As both endothelial cells and cancer cells can produce several cytokines, it was hypothesized that they may mutually influence each other. Tumor cell growth and tumor angiogenesis are increased by the recruitment of endothelial cells, contributing to the formation of the metastatic niche (8). Therefore, the modulatory effect of CX3CL1 of HCC on BMECs. CX3CL1 expression was knocked down by lentiviral transfection into BMECs to construct CX3CL1 ${ }^{\text {low }}$ BMECs; CX3CL1 ${ }^{\text {nor }}$ BMECs were used as a control. Transwell assays were to assess cell migration, and CCK- 8 assays were used to assess proliferation.HCC cells enhanced the recruitment and proliferation of $\mathrm{CX} 3 \mathrm{CL}^{\text {nor }} \mathrm{BMECs}$, but this enhancement was attenuated in CX3CL1 ${ }^{\text {low }}$ BMECs (Fig. 7A and B). It was hypothesized that soluble CX3CL1 from BMECs could stimulate HCC cells, which in turn would act on BMECs to create a vicious feedback cycle. Previous data have revealed that HCC cells induce BMECs to exhibit an aggressive phenotype, which may contribute to the formation of a bone metastatic niche (48). These data demonstrate the important regulatory role that CX3CL1 plays in the communication between HCC cells and BMECs.

To verify the effects of BMECs on the metastasis and growth of HCC cells in the spine, HCC cells were injected into the spines of mice in situ. BMECs infected with CX3CL1-silencing lentivirus were $\mathrm{CX} 3 \mathrm{CL} 1^{\text {nor }} \mathrm{BMEC}$ group while BMECs with control lentivirus were $\mathrm{CX} 3 \mathrm{CL1}^{\text {nor }} \mathrm{BMEC}$ group (Fig. S1D). In the in vivo experiments, tumor cells mixed with CX3CL1 ${ }^{\text {nor }}$ BMECs exhibited significantly larger tumors compared with those of mice injected with tumor cells mixed with CX3CL1 ${ }^{\text {low }}$ BMECs (Fig. 7C). Immunofluorescence revealed that expression of the endothelial cell marker CD31 was higher in the CX3CL1 ${ }^{\text {nor }}$ BMEC group compared with the CX3CL1 ${ }^{\text {low }}$ BMEC group (Fig. 7D). The levels of activated p-ADAM17 in endothelial cells mixed with CX3CL1 $1^{\text {low }}$ BMECs were lower compared with those in the control group (Fig. 7D). Flow cytometry was applied to detect the macrophage population. Additionally, it was also observed that the percentage and number of $\mathrm{F} 4 / 80^{+} \mathrm{CD} 11 \mathrm{~b}^{+}$macrophages were upregulated in the CX3CL1 $1^{\text {nor }}$ BMEC group compared with the CX3CL1 ${ }^{\text {low }}$ BMECs group (Fig. 7E). Flow cytometry showed that the M2 macrophage marker MRC in $\mathrm{F} 4 / 80^{+} \mathrm{CD} 11 \mathrm{~b}^{+}$cells were increased in the $\mathrm{CX} 3 \mathrm{CL}^{\text {nor }} \mathrm{BMEC}$ group compared with the CX3CL1 ${ }^{\text {low }}$ BMEC group (Fig. 7F). Taken together, the results demonstrate that BMECs are involved in the formation of a bone metastatic microenvironment, which then supports and promotes the growth of HCC cells.

\section{Discussion}

Although the communication between tumor cells and endothelial cells has been widely studied during the metastatic process of a variety of cancer types, few studies have focused on the effect of BMECs in spinal metastasis. The present study demonstrated that BMECs secrete CX3CL1 to enhance HCC metastasis. CX3CL1 was regulated by ADAM17 in BMECs and promoted HCC spinal metastasis via Src/PTK2 activation. The MHCC97H cell line was used to establish an in vitro using a nude mice model of high metastasis of HCC. Compared with $\mathrm{MHCC} 97 \mathrm{H}$ cells, the metastasis of Hep3B cells is lower (49). These two types of cells can be used to compare the differential effects of CX3CL1 on cells with different metastatic ability.

Increasing evidence has indicated that endothelial cells play a crucial role in regulating metastatic tumor cells (8). Endothelial cells display numerous aggressive invasive behaviors, such as vessel formation, the secretion of tumor-promoting cytokines and the modulation of the metastatic niche (50). BMECs play a critical role in maintaining and driving the homeostasis of hematopoiesis under normal physiological conditions (13), and alter their cytoskeleton in order to regulate and promote the adhesion of hematopoietic stem cells and to 
construct the hematopoietic microenvironment. Additionally, BMECs are a major source of secreted cytokines in the bone marrow. However, the functions and mechanisms of BMECs in spinal metastasis have not been thoroughly investigated.

Recently, increasing evidence has indicated that CX3CL1 plays an important role in regulating cell invasion, proliferation and migration of inflammatory cells and various cancer cell lines (51). For example, the expression of CX3CL1 is associated with the metastatic rate and a poor prognosis of patients with prostate cancer (19). A previous study by the authors demonstrated that CX3CL1 expression was upregulated in spinal cancellous bone (27). Therefore, the present study aimed to examine the effects and regulatory mechanisms of CX3CL1 in spinal metastasis. The expression levels of CX3CL1 in HCC spinal metastases were upregulated, and the in vitro experiments confirmed these results. Cell invasion and migration experiments demonstrated that the aggressive nature of cancer cells was decreased by the administration of a CX3CL1 neutralizing antibody. The CX3CL1 neutralizing antibody also downregulated the activation of Src/PTK2 in HCC cells. Src promoted tumor progression, the activation of which is increased in metastatic cells compared with non-metastatic cells (52). Therefore, Src activation is considered a biological marker of tumor progression (53). PP1, a Src family inhibitor, can inhibit Src activity and block downstream PTK2 signaling (54). PTK2 is a tyrosine-phosphorylated protein that influences cell invasion and migration (55). The phosphorylation of PTK2 regulates its localization at focal adhesion sites located on the plasma membrane (56). Recent studies have suggested that PTK2 is closely associated with cancer survival, metastasis and growth $(57,58)$. In several cell lines, PTK2 is often associated with integrin-related signaling at focal adhesion sites which mediate cellular migration and adhesion (59). Rho family GTPases are known regulators of cell adhesion and the inhibition of PTK2 destabilizes RHOA activity $(60,61)$. PTK2 is also a core modulator of the PIK3CA/AKT1 signaling pathway, primarily through the formation of complexes with PIK3CA (62). PIK3CA/AKT1 signaling is increased in several types of cancer, including $\mathrm{HCC}$, and promotes cancer survival and metastasis (63). In the present study, NEK2 expression was shown to be increased in cells treated with CX3CL1. NEK2 expression has been reported to be correlated with that of $\beta$-catenin in cancer (64). Taken together, these results suggest that Src/PTK2 signaling may form an essential component of the mechanisms underlying spinal metastasis in HCC.

Once metastatic cells reach the distant metastasis site, they alter the proliferation, migration and gene expression profiles of normal endothelial cells (65). Thus, whether there were any alterations to gene expression in BMECs, was assessed and it was shown that CX3CL1 was highly expressed in the cancellous bone of the spine. CX3CL1 differs from other chemokines as CX3CL1 has two functional types; a membrane-bound form and a soluble form. This dual identity of CX3CL1, both a chemokine and a receptor, allows it to function as both a chemoattractant and an adhesion molecule. The shedding of the CX3CL1 ectodomain is the process in which it is cleaved at the juxtamembrane region by metalloproteases, leading to the detachment of the extracellular region (66). This shedding process releases soluble CX3CL1 from its membrane-bound form (67). One of the primary enzymes responsible for converting CX3CL1 from a membrane-bound form to a soluble form is the proteolytically active ADAM17 (68). In the present study, ELISA analyses revealed the content of soluble form CX3CL1 in the medium was significantly reduced in BMECs transfected with ADAM17-targeting siRNA. Therefore, BMECs and HCC cells interact to enhance tumor formation in the spinal metastatic environment.

Finally, it was found that CX3CL1 promoted HCC spinal metastasis in vivo as well. Alternatively, studies have indicated that CX3CL1 treatment may improve HCC outcomes in mice by inducing antitumor immunity (69). Thus, it was hypothesized that the secreted form and membrane-bound form of CX3CL1 may exert different effects in vivo. Furthermore, the effect of CX3CL1, varying from tumor suppression to tumor enhancement, is dependent on the target tissue and the experimental model. TAMs secrete several types of inflammatory cytokines in the tumor environment (70). Activated macrophages release several molecules that may directly or indirectly affect the tumor cell behavior. Certain studies have shown that TAMs are important in the development of HCC (71). TAMs predominantly differentiate into the M2 phenotype, which is beneficial to both tumor formation and growth (72). In the present study, it was found that TAMs that were stimulated by CX3CL1 became polarized towards an M2 phenotype, thereby enhancing the invasive nature of HCC.

In conclusion, the present study demonstrated that CX3CL1 secreted from BMECs played an important role in enhancing spinal metastasis from HCC. BMECs promoted HCC invasion and migration by producing CX3CL1. ADAM17 was activated by MAPK and regulated the secretion of CX3CL1. These findings may partly explain the mechanisms underlying $\mathrm{HCC}$ spinal metastasis, and highlight potential novel targets for the treatment of HCC spinal metastasis.

\section{Acknowledgements}

Not applicable.

\section{Funding}

The present study was supported by the Natural Science Foundation of China (grant nos. 81772855 and 81572629) and the Science and Technology Commission of Shanghai Municipality (17411950302).

\section{Availability of data and materials}

All data generated or analyzed during this study are included in this published article or are available from the corresponding author on reasonable request.

\section{Authors' contributions}

JD was involved in the conception and design of the study, and in the preparation of the manuscript. CS, AH and SW were involved in conducting the experiments, and in the collection and supervision of the data. YL and HW analyzed and examined the data. BT and LJ were involved in the drafting of the manuscript and in collecting and pre-treating the clinical specimens. All authors have read and approved the manuscript. 


\section{Ethics approval and consent to participate}

All patients provided informed consent and agreed to participate in the study. The present study was approved by the Ethics Committee of Zhongshan Hospital, Fudan University (approval no. Y2014-185 and Y2019-085). All animal experiments were performed in compliance with the Guidelines for the Care and Use of Research Animals established by the Animal Ethics Committee of Zhongshan Hospital, Fudan University. The animal studies were approved by the Animal Ethics Committee of Zhongshan Hospital, Fudan University.

\section{Patient consent for publication}

Not applicable.

\section{Competing interests}

The authors declare that they have no competing interests.

\section{References}

1. Cabibbo G, Enea M, Attanasio M, Bruix J, Craxì A and Cammà C: A meta-analysis of survival rates of untreated patients in randomized clinical trials of hepatocellular carcinoma. Hepatology 51: 1274-1283, 2010

2. Makuuchi M and Sano K: Surgical treatment of HCC: Update topics. Updates Surg 63: 67-68, 2011.

3. Santini D, Pantano F, Riccardi F, Di Costanzo GG, Addeo R, Guida FM, Ceruso MS, Barni S, Bertocchi P, Marinelli S, et al: Natural history of malignant bone disease in hepatocellular carcinoma: Final results of a multicenter bone metastasis survey. PLoS One 9: e105268, 2014.

4. Choi $\mathrm{C}$ and Seong J: Predictive factors of palliative radiotherapy response and survival in patients with spinal metastases from hepatocellular carcinoma. Gut Liver 9: 94-102, 2015.

5. Kim SU, Kim DY, Park JY, Ahn SH, Nah HJ, Chon CY and Han KH: Hepatocellular carcinoma presenting with bone metastasis: Clinical characteristics and prognostic factors. J Cancer Res Clin Oncol 134: 1377-1384, 2008.

6. Hang LH, Li SN, Luo H, Shu WW, Mao ZM, Chen YF, Shi LL and Shao DH: Connexin 43 mediates CXCL12 production from spinal dorsal horn to maintain bone cancer pain in rats. Neurochem Res 41: 1200-1208, 2016.

7. Obenauf AC and Massagué J: Surviving at a distance: Organ-specific metastasis. Trends Cancer 1: 76-91, 2015.

8. Esposito M and Kang Y: Targeting tumor-stromal interactions in bone metastasis. Pharmacol Ther 141: 222-233, 2014.

9. Yousefi M, Nosrati R, Salmaninejad A, Dehghani S, Shahryari A and Saberi A: Organ-specific metastasis of breast cancer: Molecular and cellular mechanisms underlying lung metastasis. Cell Oncol (Dordr) 41: 123-140, 2018.

10. Hida K, Maishi N, Sakurai Y, Hida Y and Harashima H: Heterogeneity of tumor endothelial cells and drug delivery. Adv Drug Deliv Rev 99: 140-147, 2016.

11. Colmone A and Sipkins DA: Beyond angiogenesis: The role of endothelium in the bone marrow vascular niche. Transl Res 151: $1-9,2008$

12. Singh S, Singh R, Sharma PK, Singh UP, Rai SN, Chung LW, Cooper CR, Novakovic KR, Grizzle WE and Lillard JW Jr: Serum CXCL13 positively correlates with prostatic disease, prostate-specific antigen and mediates prostate cancer cell invasion, integrin clustering and cell adhesion. Cancer Lett 283: 29-35, 2009.

13. Kopp HG, Avecilla ST, Hooper AT and Rafii S: The bone marrow vascular niche: Home of HSC differentiation and mobilization. Physiology (Bethesda) 20: 349-356, 2005.

14. Li WM, Huang WQ, Huang YH, Jiang DZ and Wang QR: Positive and negative hematopoietic cytokines produced by bone marrow endothelial cells. Cytokine 12: 1017-1023, 2000.
15. Bade-Döding C, Göttmann W, Baigger A, Farren M, Lee KP, Blasczyk R and Huyton T: Autocrine GM-CSF transcription in the leukemic progenitor cell line KGla is mediated by the transcription factor ETS1 and is negatively regulated through SECTM1 mediated ligation of CD7. Biochim Biophys Acta 1840: 1004-1013, 2014

16. Lavi N, Kessler O, Ziv K, Nir-Zvi I, Mumblat Y, Eiza N, Paran Y, Brenner B, Vadasz Z and Neufeld G: Semaphorin-3A inhibits multiple myeloma progression in a mouse model. Carcinogenesis 39: 1283-1291, 2018.

17. Lamanuzzi A, Saltarella I, Ferrucci A, Ria R, Ruggieri S, Racanelli V, Rao L, Annese T, Nico B, Vacca A, et al: Role of erythropoietin in the angiogenic activity of bone marrow endothelial cells of MGUS and multiple myeloma patients. Oncotarget 7: 14510-14521, 2016.

18. Griffith JW, Sokol CL and Luster AD: Chemokines and chemokine receptors: Positioning cells for host defense and immunity. Annu Rev Immunol 32: 659-702, 2014.

19. Shulby SA, Dolloff NG, Stearns ME, Meucci O and Fatatis A: CX3CR1-fractalkine expression regulates cellular mechanisms involved in adhesion, migration, and survival of human prostate cancer cells. Cancer Res 64: 4693-4698, 2004.

20. Wong HS, Jaumouillé V, Heit B, Doodnauth SA, Patel S, Huang YW, Grinstein S and Robinson LA: Cytoskeletal confinement of CX3CL1 limits its susceptibility to proteolytic cleavage by ADAM10. Mol Biol Cell 25: 3884-3899, 2014.

21. Tang J, Xiao L, Cui R, Li D, Zheng X, Zhu L, Sun H, Pan Y, Du Y and Yu X: CX3CL1 increases invasiveness and metastasis by promoting epithelial-to-mesenchymal transition through the TACE/TGF- $\alpha / E G F R$ pathway in hypoxic androgen-independent prostate cancer cells. Oncol Rep 35: 1153-1162, 2016.

22. Wei LM, Cao S, Yu WD, Liu YL and Wang JT: Overexpression of CX3CR1 is associated with cellular metastasis, proliferation and survival in gastric cancer. Oncol Rep 33: 615-624, 2015.

23. Tardáguila M, Mira E, García-Cabezas MA, Feijoo AM, Quintela-Fandino M, Azcoitia I, Lira SA and Mañes S: CX3CL1 promotes breast cancer via transactivation of the EGF pathway. Cancer Res 73: 4461-4473, 2013.

24. Yao X, Qi L, Chen X, Du J, Zhang Z and Liu S: Expression of CX3CR1 associates with cellular migration, metastasis, and prognosis in human clear cell renal cell carcinoma. Urol Oncol 32: 162-170, 2014.

25. Zheng J, Yang M, Shao J, Miao Y, Han J and Du J: Chemokine receptor CX3CR1 contributes to macrophage survival in tumor metastasis. Mol Cancer 12: 141, 2013.

26. Liang Y, Yi L, Liu P, Jiang L, Wang H, Hu A, Sun C and Dong J: CX3CL1 involves in breast cancer metastasizing to the spine via the Src/FAK signaling pathway. J Cancer 9: 3603-3612, 2018.

27. Liu $\mathrm{P}$, Liang $\mathrm{Y}$, Jiang $\mathrm{L}$, Wang $\mathrm{H}$, Wang $\mathrm{S}$ and Dong J: CX3CL1/fractalkine enhances prostate cancer spinal metastasis by activating the Src/FAK pathway. Int J Oncol 53: 1544-1556, 2018.

28. Wang R, Yu Z, Chen F, Xu H, Shen S, Chen W, Chen L, Su Q, Zhang L, Bi J, et al: miR-300 regulates the epithelial-mesenchymal transition and invasion of hepatocellular carcinoma by targeting the FAK/PI3K/AKT signaling pathway. Biomed Pharmacother 103: 1632-1642, 2018.

29. Chen JS, Huang XH, Wang Q, Chen XL, Fu XH, Tan HX, Zhang LJ, Li W and Bi J: FAK is involved in invasion and metastasis of hepatocellular carcinoma. Clin Exp Metastasis 27: 71-82, 2010.

30. Li D, Zhang Y, Zhang H, Zhan C, Li X, Ba T, Qiu Z, E F, Lv G, Zou C, et al: CADM2, as a new target of miR-10b, promotes tumor metastasis through FAK/AKT pathway in hepatocellular carcinoma. J Exp Clin Cancer Res 37: 46, 2018.

31. Zhang YL, Xing X, Cai LB, Zhu L, Yang XM, Wang YH, Yang Q, Nie HZ, Zhang ZG, Li J, et al: Integrin $\alpha 9$ suppresses hepatocellular carcinoma metastasis by Rho GTPase signaling. J Immunol Res 2018: 4602570, 2018.

32. Livak KJ and Schmittgen TD: Analysis of relative gene expression data using real-time quantitative PCR and the 2(-Delta Delta C(T)) method. Methods 25: 402-408, 2001.

33. Naito S, von Eschenbach AC, Giavazzi R and Fidler IJ: Growth and metastasis of tumor cells isolated from a human renal cell carcinoma implanted into different organs of nude mice. Cancer Res 46: 4109-4115, 1986.

34. Beyer H: The Wilcoxon, Mann and Whitney U-test - a distribution-independent statistical procedure for the comparison of 2 independent random samples. Z Arztl Fortbild (Jena) 82: 871-873, 1988 (In German). 
35. Stout MC, Narayan S, Pillet ES, Salvino JM and Campbell PM: Inhibition of CX3CR1 reduces cell motility and viability in pancreatic adenocarcinoma epithelial cells. Biochem Biophys Res Commun 495: 2264-2269, 2018

36. Ishida Y, Kimura A, Nosaka M, Kuninaka Y, Hemmi H, Sasaki I, Kaisho T, Mukaida N and Kondo T: Essential involvement of the CX3CL1-CX3CR1 axis in bleomycin-induced pulmonary fibrosis via regulation of fibrocyte and M2 macrophage migration. Sci Rep 7: 16833, 2017.

37. Jamieson-Gladney WL, Zhang Y, Fong AM, Meucci O and Fatatis A: The chemokine receptor CX(3)CR1 is directly involved in the arrest of breast cancer cells to the skeleton. Breast Cancer Res 13: R91, 2011.

38. Longo V, Brunetti O, D'Oronzo S, Ostuni C, Gatti P and Silvestris F: Bone metastases in hepatocellular carcinoma: An emerging issue. Cancer Metastasis Rev 33: 333-342, 2014.

39. Pascual J and Turner NC: Targeting the PI3-kinase pathway in triple-negative breast cancer. Ann Oncol 30: 1051-1060, 2019.

40. Huang GX, Wang Y, Su J, Zhou P, Li B, Yin LJ and Lu J: Up-regulation of Rho-associated kinase $1 / 2$ by glucocorticoids promotes migration, invasion and metastasis of melanoma. Cancer Lett 410: 1-11, 2017

41. Song LJ, Liu Q, Meng XR, Li ShL, Wang LX, Fan QX and Xuan XY: DLC-1 is an independent prognostic marker and potential therapeutic target in hepatocellular cancer. Diagn Pathol 11: 19, 2016.

42. Samarin J, Laketa V, Malz M, Roessler S, Stein I, Horwitz E Singer S, Dimou E, Cigliano A, Bissinger M, et al: PI3K/AKT/ mTOR-dependent stabilization of oncogenic far-upstream element binding proteins in hepatocellular carcinoma cells. Hepatology 63: 813-826, 2016

43. Cheng Y, Chen X, Ye L, Zhang Y, Liang J, Liu W, Zhou B, Zheng S, Huang Y, Chen G, et al: The prognostic significance of NEK2 in hepatocellular carcinoma: Evidence from a meta-analysis and retrospective cohort study. Cell Physiol Biochem 51: 2746-2759, 2018.

44. Zhang Y, Wang W, Wang Y, Huang X, Zhang Z, Chen B, Xie W, Li S, Shen S and Peng B: NEK2 promotes hepatocellular carcinoma migration and invasion through modulation of the epithelial-mesenchymal transition. Oncol Rep 39: 1023-1033, 2018.

45. De Sanctis F, Ugel S, Facciponte J and Facciabene A: The dark side of tumor-associated endothelial cells. Semin Immunol 35 $35-47,2018$.

46. Bergmeier W, Piffath CL, Cheng G, Dole VS, Zhang Y, von AndrianUHandWagnerDD:Tumornecrosisfactor-alpha-converting enzyme (ADAM17) mediates GPIbalpha shedding from platelets in vitro and in vivo. Circ Res 95: 677-683, 2004.

47. Saad MI, Alhayyani S, McLeod L, Yu L, Alanazi M, Deswaerte V, Tang K, Jarde T, Smith JA, Prodanovic Z, et al: ADAM17 selectively activates the IL-6 trans-signaling/ERK MAPK axis in KRAS-addicted lung cancer. EMBO Mol Med 11: 11, 2019.

48. Zhu H, Shao Q, Sun X, Deng Z, Yuan X, Zhou X and Ding Y: Bone marrow cells: Important role on neovascularization of hepatocellular carcinoma. J Gastroenterol Hepatol 27: 1241-1251, 2012.

49. Pan Q, Wang L, Sun HC, Liu YK, Ye SL and Tang ZY: Transcription factor activity profile of human hepatocellular carcinoma cell lines with different metastatic potentials. Zhonghua Gan Zang Bing Za Zhi 14: 37-40, 2006 (In Chinese).

50. Kusumbe AP, Ramasamy SK and Adams RH: Coupling of angiogenesis and osteogenesis by a specific vessel subtype in bone. Nature 507: 323-328, 2014.

51. Liu W, Jiang L, Bian C, Liang Y, Xing R, Yishakea M and Dong J: Role of CX3CL1 in diseases. Arch Immunol Ther Exp (Warsz) 64: 371-383, 2016

52. Guarino M: Src signaling in cancer invasion. J Cell Physiol 223 $14-26,2010$

53. Aligayer H, Boyd DD, Heiss MM, Abdalla EK, Curley SA and Gallick GE: Activation of Src kinase in primary colorecta carcinoma: An indicator of poor clinical prognosis. Cancer 94: 344-351, 2002.
54. Van Slambrouck S, Grijelmo C, De Wever O, Bruyneel E, Emami S, Gespach C and Steelant WF: Activation of the FAK-src molecular scaffolds and p130Cas-JNK signaling cascades by alpha1-integrins during colon cancer cell invasion. Int J Oncol 31 : 1501-1508, 2007

55. Yoon H, Dehart JP, Murphy JM and Lim ST: Understanding the roles of FAK in cancer: Inhibitors, genetic models, and new insights. J Histochem Cytochem 63: 114-128, 2015.

56. Hu YL, Lu S, Szeto KW, Sun J, Wang Y, Lasheras JC and Chien S: FAK and paxillin dynamics at focal adhesions in the protrusions of migrating cells. Sci Rep 4: 6024, 2014.

57. Sulzmaier FJ, Jean C and Schlaepfer DD: FAK in cancer: Mechanistic findings and clinical applications. Nat Rev Cancer 14: 598-610, 2014.

58. Jeong KY: Inhibiting focal adhesion kinase: A potential target for enhancing therapeutic efficacy in colorectal cancer therapy. World J Gastrointest Oncol 10: 290-292, 2018.

59. Golubovskaya VM: Focal adhesion kinase as a cancer therapy target. Anticancer Agents Med Chem 10: 735-741, 2010

60. Schmidt TT, Tauseef M, Yue L, Bonini MG, Gothert J, Shen TL, Guan JL, Predescu S, Sadikot R and Mehta D: Conditional deletion of FAK in mice endothelium disrupts lung vascular barrier function due to destabilization of RhoA and Racl activities. Am J Physiol Lung Cell Mol Physiol 305: L291-L300, 2013.

61. Thiery JP: Epithelial-mesenchymal transitions in tumour progression. Nat Rev Cancer 2: 442-454, 2002.

62. Luo J, Yao JF, Deng XF, Zheng XD, Jia M, Wang YQ, Huang Y and Zhu JH: 14, 15-EET induces breast cancer cell EMT and cisplatin resistance by up-regulating integrin $\alpha v \beta 3$ and activating FAK/PI3K/AKT signaling. J Exp Clin Cancer Res 37: 23, 2018.

63. Zheng H, Yang Y, Hong YG, Wang MC, Yuan SX, Wang ZG, Bi FR, Hao LQ, Yan HL and Zhou WP: Tropomodulin 3 modulates EGFR-PI3K-AKT signaling to drive hepatocellular carcinoma metastasis. Mol Carcinog 58: 1897-1907, 2019.

64. Neal CP, Fry AM, Moreman C, McGregor A, Garcea G, Berry DP and Manson MM: Overexpression of the Nek2 kinase in colorectal cancer correlates with beta-catenin relocalization and shortened cancer-specific survival. J Surg Oncol 110: 828-838, 2014.

65. Akino T, Hida K, Hida Y, Tsuchiya K, Freedman D, Muraki C, Ohga N, Matsuda K, Akiyama K, Harabayashi $\mathrm{T}$, et al: Cytogenetic abnormalities of tumor-associated endothelial cells in human malignant tumors. Am J Pathol 175: 2657-2667, 2009.

66. Montes de Oca-B P: Ectdomain shedding and regulated intracellular proteolysis in the central nervous system. Cent Nerv Syst Agents Med Chem 10: 337-359, 2010.

67. Dreymueller D, Martin C, Kogel T, Pruessmeyer J, Hess FM, Horiuchi K, Uhlig S and Ludwig A: Lung endothelial ADAM17 regulates the acute inflammatory response to lipopolysaccharide. EMBO Mol Med 4: 412-423, 2012.

68. Tsou CL, Haskell CA and Charo IF: Tumor necrosis factor-alpha-converting enzyme mediates the inducible cleavage of fractalkine. J Biol Chem 276: 44622-44626, 2001.

69. Tang L, Hu HD, Hu P, Lan YH, Peng ML, Chen M and Ren H: Gene therapy with CX3CL1/Fractalkine induces antitumor immunity to regress effectively mouse hepatocellular carcinoma. Gene Ther 14: 1226-1234, 2007.

70. Rades D, Stalpers LJ, Hulshof MC, Borgmann K, Karstens JH, Koning CC and Alberti W: Comparison of 1x8 Gy and 10x3 Gy for functional outcome in patients with metastatic spinal cord compression. Int J Radiat Oncol Biol Phys 62: 514-518, 2005.

71. Li Z, Wu T, Zheng B and Chen L: Individualized precision treatment: Targeting TAM in HCC. Cancer Lett 458: 86-91, 2019.

72. Atanasov G, Dino K, Schierle K, Dietel C, Aust G, Pratschke J, Seehofer D, Schmelzle M and Hau HM: Immunologic cellular characteristics of the tumour microenvironment of hepatocellular carcinoma drive patient outcomes. World J Surg Oncol 17: 97 , 2019. International (CC BY-NC-ND 4.0) License. 